TRANSACTIONS OF THE

AMERICAN MATHEMATICAL SOCIETY

Volume 350, Number 12, December 1998, Pages 4931-4953

S $0002-9947(98) 02020-0$

\title{
THE SINGULAR LIMIT OF A VECTOR-VALUED REACTION-DIFFUSION PROCESS
}

\author{
LIA BRONSARD AND BARBARA STOTH
}

\begin{abstract}
We study the asymptotic behaviour of the solution to the vectorvalued reaction-diffusion equation

$$
\varepsilon \partial_{t} \varphi-\varepsilon \Delta \varphi+\frac{1}{\varepsilon} \tilde{W}_{, \varphi}(\varphi)=0 \quad \text { in } \Omega_{T},
$$

where $\varphi_{\varepsilon}=\varphi: \Omega_{T}:=(0, T) \times \Omega \longrightarrow \mathbf{R}^{2}$. We assume that the the potential $\tilde{W}$ depends only on the modulus of $\varphi$ and vanishes along two concentric circles. We present a priori estimates for the solution $\varphi$, and, in the spatially radially symmetric case, we show rigorously that in the singular limit as $\varepsilon \rightarrow 0$, two phases are created. The interface separating the bulk phases evolves by its mean curvature, while $\varphi$ evolves according to a harmonic map flow on the respective circles, coupled across the interfaces by a jump condition in the gradient.
\end{abstract}

\section{INTRODUCTION}

In this paper, we study the singular limit of the vector-valued reaction-diffusion equation for $\varphi_{\varepsilon}=\varphi: \Omega_{T}:=(0, T) \times \Omega \longrightarrow \mathbf{R}^{2}$,

$$
\varepsilon \partial_{t} \varphi-\varepsilon \triangle \varphi+\frac{1}{\varepsilon} \tilde{W}_{, \varphi}(\varphi)=0 \quad \text { in } \Omega_{T},
$$

with Neumann condition on $(\partial \Omega)_{T}$, where $\Omega$ is a smooth bounded domain in $\mathbb{R}^{n}, n \geq$ 2. We assume that the potential $\tilde{W}$ depends only on the modulus of $\varphi$, that it is smooth, that it vanishes along two circles and that it is positive elsewhere. A typical example is given by $\tilde{W}(\varphi)=(|\varphi|-a)^{2}(b-|\varphi|)^{2}$. In the singular limit as $\varepsilon \rightarrow 0$, one expects the domain $\Omega$ to be divided in bulk regions where $|\varphi|$ is close to $a$ or $b$. We present a priori estimates for the solution $\varphi$ and, in the radial case, we prove that the interfaces separating the bulk regions evolve normally according to their curvature as $\varepsilon \rightarrow 0$. Moreover we establish the diffusion equation satisfied by $\varphi$ in the bulk regions as $\varepsilon \rightarrow 0$, coupled across the interfaces by a jump condition in the gradient. This jump condition is new and was not previously derived in the formal asymptotic analysis of this system (cf. [RSK]).

Rubinstein, Sternberg and Keller [RSK] introduced this vector-valued reactiondiffusion equation for $\varphi \in \mathbf{R}^{N}, N \geq 2$, to model certain chemical reactions. Indeed in chemical reactions, the (nonconserved) order parameter $\varphi$ represents a vector of concentration of reactants, and the law of mass action leads to the above reactiondiffusion equation with the potential $\tilde{W}$ vanishing on one or more manifolds. In their paper [RSK], Rubinstein, Sternberg and Keller presented formal asymptotic

Received by the editors November 17, 1995 and, in revised form, October 15, 1996.

1991 Mathematics Subject Classification. Primary 35B25, 35K57. 
expansions when the potential vanishes on two manifolds. Convergence proofs were given in $[\mathrm{RSK}]$ and $[\mathrm{C}]$ when the potential vanishes on one sphere, and in [CS] when it vanishes on a general connected manifold. In fact, Chen [C] and Chen-Struwe [CS] used this parabolic system to study existence and singularity formation of the harmonic map flow when the target manifold is respectively a sphere and a general connected manifold.

We study rigorously the radial case with $\varphi \in \mathbf{R}^{2}$, and with a potential that only depends on the modulus $|\varphi|$ and vanishes on two concentric spheres. We always assume that the energy is initially bounded (cf. (A1)). We choose initial data that lie strictly in one half plane (cf. (A2), (A3) and (A4)). We may then represent $\varphi$ through $u(\cos f, \sin f)$, where $u$ is the modulus of $\varphi$ and $f$ is the polar angle, and rewrite the problem for $\varphi$ as a system of equations for $u$ and $f$ (cf. Section 2 , equations (E2) and (E3)). By standard techniques a subsequence of $u=u_{\varepsilon}$ converges to a limit $u_{0}$ (cf. Remark 3.2). We also show that a subsequence of $f=f_{\varepsilon}$ converges to a limit $f_{0}$ (cf. Lemma 3.5 ).

The results of Rubinstein, Sternberg and Keller [RSK] suggest that the interfaces $\Gamma$ separating the regions $\Omega_{a}$ and $\Omega_{b}$, where $u_{0}$ is either $a$ or $b$, evolve normally by their curvature. We prove this result in the radial case by presenting the convergence of (E2) to a weak formulation of curvature flow (cf. Section 5, Proposition 5.2 and Theorem 5.5). In addition, the results in [RSK] suggest that the limit $f_{0}$ of the angle $f$ satisfies a harmonic map flow in the bulk phases. We rigorously verify this assertion in the radial case by passing to the limit in the equation (E3) satisfied by $f$ (cf. Section 6, Theorem 6.3). We show that $f_{0}$ is a generalized solution of

$$
u_{0}^{2} \partial_{t} f_{0}-\operatorname{div}\left(u_{0}^{2} \nabla f_{0}\right)=0 .
$$

This equation couples the harmonic flow $\partial_{t} f_{0}-\triangle f_{0}=0$ in the bulk regions $\Omega_{a}$ and $\Omega_{b}$ to the jump condition

$$
\left[u_{0}^{2} \nabla f_{0}\right]_{\mid \Gamma} \cdot \nu=0
$$

on the interfaces $\Gamma$. This condition, which was not explicitly derived in [RSK], arises as a byproduct of our analysis. Of course, with the benefit of hindsight, one can formally derive this jump condition, and we include a brief description of this formal derivation in Remark 6.5 in the context of [RSK].

To prove these results, we extend the energy method developed in [BSt], which is based on ideas introduced in $[\mathrm{BK} 1,2]$ and $[\mathrm{St} 1,2]$ to treat scalar-valued reactiondiffusion equations. One advantage of the energy method is that it does not rely on comparison principles (e.g. the maximum principle), and in this paper we show that we can extend it to treat systems. The new feature of the method, once specialized to (E1), is the derivation of the necessary a priori estimates on the solutions $u$ and $f$ (cf. Section 3, Lemma 3.4, Lemma 3.6 and Lemma 3.8, and Section 4, Proposition 4.1 ), but the underlying principles of the method remain unaltered. In particular we prove that $u_{\varepsilon}$ is well approximated by a stationary wave solution as in the case of the scalar Allen-Cahn equation. Our main result is contained in the following theorem.

Theorem. Let $\tilde{W}$ be a smooth double well potential satisfying (W1) and (W2) of Section 2. Let $\varphi_{\varepsilon}^{0}=u_{\varepsilon}^{0}\left(\cos f_{\varepsilon}^{0}, \sin f_{\varepsilon}^{0}\right)$ be a smooth, radially symmetric function satisfying (A1)-(A4) of Section 3, and for any $\varepsilon>0$ let $\varphi_{\varepsilon}=u_{\varepsilon}\left(\cos f_{\varepsilon}\right.$, $\left.\sin f_{\varepsilon}\right)$ be the smooth solution of equation (E1) of Section 2 with Neumann condition on the spatial boundary and initial values $\varphi_{\varepsilon}^{0}$. 
Then there exist a subsequence $\varepsilon \rightarrow 0$ and limits $u_{0} \in L^{\infty}(0, T ; B V(\Omega)) \cap B V\left(\Omega_{T}\right)$ and $f_{0} \in L^{\infty}\left(\Omega_{T}\right) \cap L^{2}\left(0, T ; H^{1,2}(\Omega)\right)$, such that $u_{\varepsilon} \rightarrow u_{0}$ in $L^{1}\left(\Omega_{T}\right)$ and $f_{\varepsilon} \rightarrow f_{0}$ in $L^{1}\left(\Omega_{T}\right)$.

The order parameter $u_{0}$ takes only the values $a$ and $b$ almost everywhere in $\Omega_{T}$, and the free boundary $\partial\left\{u_{0}=a\right\}$ consists of a collection of graphs $R^{j}:\left[t^{j}, T^{j}\right) \rightarrow$ $(0,1)$ of class $H_{\text {loc }}^{1,2}\left(\left[t^{j}, T^{j}\right)\right)$ that evolve according to their curvature:

$$
\frac{d}{d t} R^{j}=-\frac{n-1}{R^{j}} \quad \text { in }\left(t^{j}, T^{j}\right) .
$$

The limit angle $f_{0}$ satisfies a harmonic map flow in bulk, a jump condition on the interface $\partial\left\{u_{0}=a\right\}$ and a Neumann condition on the boundary:

$$
u_{0}^{2} \partial_{t} f_{0}-\operatorname{div}\left(u_{0}^{2} \nabla f_{0}\right)=0 \quad \text { in the sense of Theorem 6.3. }
$$

Next, we remark on the hypothesis of radial symmetry, which is essential for our approach. At the present time there are few analytical methods for treating nonradial problems, and in fact we know of no method which has been used for rigorous analysis of singularly perturbed systems such as (E1). Moreover, our rigorous radial results already give a more profound understanding of the formal asymptotic results in [RSK]. In fact, by interpreting the limiting problem in an analytically rigorous way (as a weak solution) we were able to identify the correct jump condition, which their formal analysis did not reveal.

Finally, if the potential $W$ vanishes on two manifolds but does not have the symmetry assumed here, the results in [RSK] suggest that the interfaces will still evolve by their mean curvature, while the bulk flows will be given by harmonic map flows taking values in each manifold. However it is not known (even formally) how the flows in the bulk regions are coupled across the interfaces. We expect the following: define the distance between the two manifolds as in Sternberg [S1]. It is given by the minimal energy of stationary waves connecting pair of points on the manifolds (see [S2], [BR]). If there is exactly one stationary wave of minimal energy, we expect that the boundary values of the bulk flows at the interface will coincide with the endpoints of this stationary wave. On the other hand, if the distance between the manifolds is attained at a continuum of points (as in the radially symmetric case), then we expect that there will also be a jump condition in the gradient across the interface. In order to prove this result, the order parameter $\varphi$ must be decomposed into a quantity that determines the interface, and another quantity that determines the bulk flow. In the present paper this is achieved by representing $\varphi$ as $u e^{i f}$.

\section{Formulation of the Problem}

Let $\Omega \subset \mathbb{R}^{n}$ be a bounded domain with smooth boundary, $T>0$ and $\Omega_{T}:=$ $(0, T) \times \Omega$.

We always assume that

$$
\varphi_{\varepsilon}=\varphi: \Omega_{T} \longrightarrow \mathbf{R}^{2}
$$

is a smooth function that satisfies

$$
\varepsilon \partial_{t} \varphi-\varepsilon \triangle \varphi+\frac{1}{\varepsilon} \tilde{W}_{, \varphi}(\varphi)=0 \quad \text { in } \Omega_{T},
$$

with Neumann condition on $(\partial \Omega)_{T}$ and with initial values $\varphi(0, \cdot)=\varphi^{0}=\varphi_{\varepsilon}^{0}$, that we assume to lie strictly in the right half plane. 
We make the following assumptions on the potential $\tilde{W}$ : (W1)

$$
\tilde{W}(\varphi)=W(|\varphi|),
$$

where $|\cdot|$ is the euclidean norm and $W:(0, \infty) \rightarrow[0, \infty)$ is a smooth function that vanishes exactly at $a$ and $b$;

(W2) $\quad W^{\prime}$ vanishes exactly at $a, b$ and some $d \in(a, b)$.

A typical example is $W(\lambda)=(\lambda-a)^{2}(b-\lambda)^{2}$, in which case $d=(a+b) / 2$.

\section{Lemma 2.1.}

$$
\max _{\overline{\Omega_{T}}}|\varphi| \leq \max \left(b, \sup _{\bar{\Omega}}\left|\varphi^{0}\right|\right)
$$

If $\varphi_{1}^{0}>0$ in $\bar{\Omega}$, then

$$
\min _{\overline{\Omega_{T}}} \frac{\varphi_{1}}{|\varphi|} \geq \min _{\bar{\Omega}} \frac{\varphi_{1}^{0}}{\left|\varphi^{0}\right|}
$$

and

$$
\min _{\overline{\Omega_{T}}} \varphi_{1} \geq \min _{\bar{\Omega}}\left(\varphi_{1}^{0}, \frac{a \varphi_{1}^{0}}{\left|\varphi^{0}\right|}\right)
$$

Proof. We again introduce $u:=|\varphi|$ and $e:=\frac{\varphi}{|\varphi|}$ (then $e_{1}=\frac{\varphi_{1}}{|\varphi|}$ ), as long as $|\varphi|$ does not attain zero. Then $u$ satisfies the differential equation

$$
\partial_{t} u-\triangle u+\frac{1}{\varepsilon^{2}} W^{\prime}(u)=-u|\nabla e|^{2},
$$

and $e$ satisfies

$$
u^{2} \partial_{t} e-\operatorname{div}\left(u^{2} \nabla e\right)=e u^{2}|\nabla e|^{2} .
$$

The first claim now follows from the maximum principle for the first equation. The second follows because

$$
M:=\left\{(u, e) \mid e_{1} \geq c_{0} \text { and } \varphi_{1}=u e_{1} \geq c_{1}\right\}
$$

is an invariant region for the above flow. Here $c_{0}>0$ and $0<c_{1}<a c_{0}$, where $a$ is the first minimum of $W$ (cf. W2). As a consequence $u=|\varphi|$ never attains zero, and thus $e$ is a well defined quantity in all of $\Omega_{T}$. In addition, $\varphi$ ranges stricty in the right half plane.

Remark and Formulation 2.2. Assume that the initial data satisfy $\varphi_{1}^{0}>0$ in $\bar{\Omega}$.

Since by assumption and as a consequence of Lemma $2.1 \varphi$ remains strictly in the right half plane, it follows that $u_{\varepsilon}=u=|\varphi|$ and $e_{\varepsilon}=e=\frac{\varphi}{|\varphi|}$ are well defined and $e$ only ranges in the right half-sphere.

Thus there exists a smooth lift $f_{\varepsilon}=f: \bar{\Omega}_{T} \rightarrow\left(-\frac{\pi}{2}, \frac{\pi}{2}\right)$, such that

$$
\varphi=u(\cos f, \sin f) \text {. }
$$

By direct calculation, using the fact that $\partial_{i} e \cdot e=0$, we get

$$
\nabla u \cdot \nabla e=(\nabla u \cdot \nabla f) \cdot e^{\perp} \quad \text { and } \quad \triangle e=-|\nabla f|^{2} e+\triangle f e^{\perp} .
$$

The terms in the equation for $\varphi$ become

$$
\begin{aligned}
\partial_{t} \varphi & =\partial_{t} u e+u \partial_{t} e, \\
\triangle \varphi & =\triangle u e+2 \nabla u \cdot \nabla e+u \triangle e, \\
\tilde{W}_{, \varphi}(\varphi) & =W^{\prime}(u) e .
\end{aligned}
$$


Taking the inner product of equation (E1) with $e$ and $u e^{\perp}$ resp., we find for $(t, x) \in$ $\Omega_{T}$ the system

$$
\begin{gathered}
\varepsilon \partial_{t} u-\varepsilon \Delta u+\varepsilon u|\nabla f|^{2}+\frac{1}{\varepsilon} W^{\prime}(u)=0, \\
u^{2} \partial_{t} f-\operatorname{div}\left(u^{2} \nabla f\right)=0,
\end{gathered}
$$

together with Neumann conditions for $u$ and $f$ on the boundary $(\partial \Omega)_{T_{\varepsilon}}$ and initial conditions $u_{\varepsilon}^{0}=u^{0}:=\left|\varphi^{0}\right|$ and $f_{\varepsilon}^{0}=f^{0}$, where $u^{0}$ and $f^{0}$ are defined through

$$
\varphi^{0}=u^{0}\left(\cos f^{0}, \sin f^{0}\right) .
$$

\section{Estimates}

We define the energy

$$
E_{\varepsilon}(\varphi):=\int_{\Omega}\left(\frac{\varepsilon}{2}|\nabla \varphi|^{2}+\frac{1}{\varepsilon} W(|\varphi|)\right) d x
$$

where we adopt the notation $|\nabla \varphi|^{2}=\sum_{i}\left|\nabla \varphi_{i}\right|^{2}$ and $\left|\partial_{t} \varphi\right|^{2}=\sum_{i}\left|\partial_{t} \varphi_{i}\right|^{2}$.

Assumptions on the initial data. We assume that the initial data $\varphi_{\varepsilon}^{0}$ are given in the form $u_{\varepsilon}^{0}\left(\cos f_{\varepsilon}^{0}, \sin f_{\varepsilon}^{0}\right)$ and that there exist $C_{0}$ and $c_{0}$, such that for all $\varepsilon$,

and

$$
\begin{gathered}
E_{\varepsilon}\left(\varphi_{\varepsilon}^{0}\right) \leq C_{0}, \\
\inf _{\bar{\Omega}} u_{\varepsilon}^{0}(x) \geq c_{0},
\end{gathered}
$$

$$
\sup _{\Omega} u_{\varepsilon}^{0}(x) \leq C_{0}
$$

$$
\sup _{\bar{\Omega}}\left|f_{\varepsilon}^{0}(x)\right|<\frac{\pi}{2}
$$

Throughout this paper we will denote by $C$ any constant that only depends on $n$, $\Omega, T, W$ as well as on $c_{0}$ and $C_{0}$.

Remark 3.1. Since $E_{\varepsilon}$ is a Lyapunov functional for equation (E1), we conclude that (A1) is satisfied also at any positive time $t \leq T$ and

(energy bound)

$$
\varepsilon \int_{t_{1}}^{t_{2}} \int_{\Omega}\left|\partial_{t} \varphi_{\varepsilon}\right|^{2} d x d t+\left.E_{\varepsilon}\left(\varphi_{\varepsilon}\right)\right|_{t_{1}} ^{t_{2}}=0
$$

for all $0 \leq t_{1} \leq t_{2} \leq T$. We note that in terms of $u_{\varepsilon}$ and $f_{\varepsilon}$ the energy becomes

$$
E_{\varepsilon}\left(\varphi_{\varepsilon}\right)=\int_{\Omega}\left(\frac{\varepsilon}{2}\left(\left|\nabla u_{\varepsilon}\right|^{2}+u_{\varepsilon}^{2}\left|\nabla f_{\varepsilon}\right|^{2}\right)+\frac{1}{\varepsilon} W\left(u_{\varepsilon}\right)\right) d x
$$

and

$$
\int_{\Omega}\left|\partial_{t} \varphi_{\varepsilon}\right|^{2} d x=\int_{\Omega}\left(\left|\partial_{t} u_{\varepsilon}\right|^{2}+u_{\varepsilon}^{2}\left|\partial_{t} f_{\varepsilon}\right|^{2}\right) d x .
$$

Lemma 2.1 implies that (A2)-(A4) are satisfied for any positive time $t \leq T$. Thus we have uniform $L^{\infty}$-bounds for $E_{\varepsilon}\left(\varphi_{\varepsilon}\right), u_{\varepsilon}, \frac{1}{u_{\varepsilon}}$ and $f_{\varepsilon}$. 
Remark 3.2 (Compactness of $u_{\varepsilon}$ ). The energy bound implies that

$$
\int_{0}^{T} \int_{\Omega}\left|\partial_{t} g\left(u_{\varepsilon}\right)\right| d x d t \quad \text { and } \quad \sup _{t} \int_{\Omega}\left|\nabla g\left(u_{\varepsilon}\right)\right| d x
$$

are uniformly bounded, where $g$ is defined by $g^{\prime}(\lambda)=\sqrt{2 W(\lambda)}$ and $g(0)=0$. (This follows from $\left(\partial_{t}, \nabla\right) g\left(u_{\varepsilon}\right)=\sqrt{2 W\left(u_{\varepsilon}\right)}\left(\partial_{t}, \nabla\right) u_{\varepsilon}$ and the Cauchy-Schwartz inequality.) Thus we may select a subsequence $\varepsilon \rightarrow 0$, such that

$$
\begin{array}{cl}
u_{\varepsilon} \longrightarrow u_{0} & \text { pointwise and strongly in } L^{1}\left(\Omega_{T}\right), \\
\left(\partial_{t}, \nabla\right) g\left(u_{\varepsilon}\right) \stackrel{*}{\longrightarrow} g_{0}\left(\partial_{t}, \nabla\right) u_{0} & \text { in the weak * topology of }\left(C^{0}\left(\Omega_{T}\right)\right)^{\prime},
\end{array}
$$

where $g_{0}:=g(b)-g(a)$.

We restrict any further discussion to this particular subsequence.

Lemma 3.3. For any $T \geq t_{2}>t_{1} \geq 0$

$$
\left.\frac{\varepsilon}{2} \int_{\Omega}\left|\partial_{t} \varphi_{\varepsilon}\right|^{2}(\cdot, x) d x\right|_{t_{1}} ^{t_{2}} \leq\left.\frac{C}{\varepsilon^{2}} E_{\varepsilon}\left(\varphi_{\varepsilon}\right)\right|_{t_{2}} ^{t_{1}}
$$

Proof. We differentiate the equation (E1) for $\varphi_{\varepsilon}$ with respect to $t$, multiply by $\partial_{t} \varphi_{\varepsilon}$ and integrate:

$$
\begin{aligned}
\left.\frac{\varepsilon}{2} \int_{\Omega}\left|\partial_{t} \varphi_{\varepsilon}\right|^{2} d x\right|_{t_{1}} ^{t_{2}} & +\varepsilon \int_{t_{1}}^{t_{2}} \int_{\Omega}\left|\partial_{t} \nabla \varphi_{\varepsilon}\right|^{2} d x d t \\
& =-\frac{1}{\varepsilon} \int_{t_{1}}^{t_{2}} \int_{\Omega}\left(W^{\prime \prime}\left(u_{\varepsilon}\right)\left|\partial_{t} u_{\varepsilon}\right|^{2}+u_{\varepsilon} W^{\prime}\left(u_{\varepsilon}\right)\left|\partial_{t} f_{\varepsilon}\right|^{2}\right) d x d t
\end{aligned}
$$

Then we use the energy bound and the boundedness of $u_{\varepsilon}$ and its inverse to conclude.

\section{Lemma 3.4.}

$$
\int_{0}^{T} \int_{\Omega} u_{\varepsilon}^{2}\left|\nabla f_{\varepsilon}\right|^{2} d x d t \leq C .
$$

Proof. Since $g\left(u_{\varepsilon}\right)$ is uniformly bounded in $B V\left(\Omega_{T}\right)$ and $g$ is monotone increasing, we conclude that for some $\lambda_{\varepsilon} \in\left(a+\frac{b-a}{4}, b-\frac{b-a}{4}\right)$ the step function

$$
\chi_{\varepsilon}:= \begin{cases}a, & \text { if } u_{\varepsilon} \leq \lambda_{\varepsilon}, \\ b, & \text { if } u_{\varepsilon}>\lambda_{\varepsilon},\end{cases}
$$

is uniformly bounded in $B V\left(\Omega_{T}\right)$. We multiply the differential equation (E3) by $f_{\varepsilon}$ and integrate:

$$
\int_{0}^{T} \int_{\Omega} u_{\varepsilon}^{2}\left|\nabla f_{\varepsilon}\right|^{2} d x d t=-\frac{1}{2} \int_{0}^{T} \int_{\Omega} u_{\varepsilon}^{2} \partial_{t} f_{\varepsilon}^{2} d x d t
$$

We write $u_{\varepsilon}^{2}$ in the term on the right hand side as $\chi_{\varepsilon}^{2}+\left(u_{\varepsilon}^{2}-\chi_{\varepsilon}^{2}\right)$. We integrate the $\chi_{\varepsilon}^{2}$-term by parts and find that the result is bounded as a consequence of the estimates of Remark 3.1. Moreover we note that

$$
\left|u_{\varepsilon}^{2}-\chi_{\varepsilon}^{2}\right| \leq C\left|u_{\varepsilon}-\chi_{\varepsilon}\right| \leq C \sqrt{W\left(u_{\varepsilon}\right)} .
$$


Thus the term with the difference $u_{\varepsilon}^{2}-\chi_{\varepsilon}^{2}$ can be estimated by

$$
C\left(\frac{1}{\varepsilon} \int_{0}^{T} \int_{\Omega} W\left(u_{\varepsilon}\right) d x d t\right)^{\frac{1}{2}}\left(\varepsilon \int_{0}^{T} \int_{\Omega}\left|\partial_{t} f_{\varepsilon}\right|^{2} d x d t\right)^{\frac{1}{2}}
$$

and is therefore bounded. This yields the result.

Lemma 3.5 (Compactness for $f_{\varepsilon}$ ). We may select a further subsequence such that

$$
\begin{gathered}
f_{\varepsilon} \longrightarrow f_{0} \quad \text { pointwise and in } L^{1}\left(\Omega_{T}\right), \\
\nabla f_{\varepsilon} \longrightarrow \nabla f_{0} \quad \text { weakly in } L^{2}\left(\Omega_{T}\right) .
\end{gathered}
$$

Proof. We use the same auxiliary function $\chi_{\varepsilon}$ as in the proof of Lemma 3.4. We approximate $\chi_{\varepsilon}$ by a smooth function $\tilde{\chi}_{\varepsilon}$ such that

$$
\int_{0}^{T} \int_{\Omega}\left|\left(\partial_{t}, \nabla\right) \tilde{\chi}_{\varepsilon}\right| d x d t \leq 2 \int_{0}^{T} \int_{\Omega}\left|\left(\partial_{t}, \nabla\right) \chi_{\varepsilon}\right| d x d t
$$

and

$$
\int_{0}^{T} \int_{\Omega}\left|\chi_{\varepsilon}-\tilde{\chi}_{\varepsilon}\right| d x d t \leq \varepsilon
$$

The differential equation for $f_{\varepsilon}$ implies that

$$
\partial_{t}\left(\tilde{\chi}_{\varepsilon}^{2} f_{\varepsilon}\right)=\partial_{t} \tilde{\chi}_{\varepsilon}^{2} f_{\varepsilon}+\left(\tilde{\chi}_{\varepsilon}^{2}-u_{\varepsilon}^{2}\right) \partial_{t} f_{\varepsilon}+\operatorname{div}\left(u_{\varepsilon}^{2} \nabla f_{\varepsilon}\right) .
$$

In order to prove the compactness, we follow ideas of Alt and Luckhaus [AL]. We use the fact that we have good control of $\partial_{t}\left(\tilde{\chi}_{\varepsilon}^{2} f_{\varepsilon}\right)$ in some weak norm, and good control of $\nabla\left(\tilde{\chi}_{\varepsilon}^{2} f_{\varepsilon}\right)$ in some strong norm.

We first show that for any $\max (2, n)<p<\infty$

$$
\int_{0}^{T-h}\left\|\left(\tilde{\chi}_{\varepsilon}^{2} f_{\varepsilon}\right)(t+h, \cdot)-\left(\tilde{\chi}_{\varepsilon}^{2} f_{\varepsilon}\right)(t, \cdot)\right\|_{W^{-1, p^{\prime}}(\Omega)} d t \leq C \cdot h .
$$

For this we note that the term in question is equal to

$$
\int_{0}^{T-h} \sup _{\|\zeta\|_{W_{0}^{1, p}(\Omega)} \leq 1} \int_{\Omega} \int_{t}^{t+h}\left(\left(\partial_{t} \tilde{\chi}_{\varepsilon}^{2} f_{\varepsilon}+\left(\tilde{\chi}_{\varepsilon}^{2}-u_{\varepsilon}^{2}\right) \partial_{t} f_{\varepsilon}\right) \zeta-u_{\varepsilon}^{2} \nabla f_{\varepsilon} \cdot \nabla \zeta\right) d x d \tau d t .
$$

Since $p>n$ and $p>2$, we may estimate this by

$$
\int_{0}^{T-h} \int_{t}^{t+h} \int_{\Omega}\left(\left|\partial_{t} \tilde{\chi}_{\varepsilon}^{2} f_{\varepsilon}\right|+\left|\left(\tilde{\chi}_{\varepsilon}^{2}-u_{\varepsilon}^{2}\right) \partial_{t} f_{\varepsilon}\right|+u_{\varepsilon}^{4}\left|\nabla f_{\varepsilon}\right|^{2}\right) d x d \tau d t .
$$

We then proceed as in the proof of Lemma 3.4, using the estimates on $\partial_{t} \tilde{\chi}_{\varepsilon}$ and on $f_{\varepsilon}$ to obtain $(*)$.

Next we use the following interpolation inequality: Assume that $1<p<\infty$. Then there exists some $C>0$ such that for any smooth function $w$ and any positive $\rho$

$$
\|w\|_{L^{1}(\Omega)} \leq C\left(\rho\|\nabla w\|_{L^{1}(\Omega)}+\frac{1}{\rho}\|w\|_{W^{-1, p^{\prime}}(\Omega)}+\rho\|w\|_{L^{\infty}(\Omega)}\right) .
$$

For a proof of this interpolation inequality we refer to [Lu]. 
We apply this to $w=\left(\tilde{\chi}_{\varepsilon}^{2} f_{\varepsilon}\right)(t+h, \cdot)-\left(\tilde{\chi}_{\varepsilon}^{2} f_{\varepsilon}\right)(t, \cdot)$, integrate in time and use the above estimate $(*)$ as well as the bound on $\nabla \tilde{\chi}_{\varepsilon}$ to conclude that

$$
\int_{0}^{T-h} \int_{\Omega}\left|\left(\tilde{\chi}_{\varepsilon}^{2} f_{\varepsilon}\right)(t+h, x)-\left(\tilde{\chi}_{\varepsilon}^{2} f_{\varepsilon}\right)(t, x)\right| d x d t \leq C\left(\rho+\frac{h}{\rho}\right) .
$$

We choose $\rho=\sqrt{h}$.

We now may use the Kolmogorov theorem to deduce that $\tilde{\chi}_{\varepsilon}^{2} f_{\varepsilon}$ is precompact in $L^{1}$, and thus that there exists a further subsequence such that $\tilde{\chi}_{\varepsilon}^{2} f_{\varepsilon}$ converges in $L^{1}$ and pointwise. Since $\tilde{\chi}_{\varepsilon}$ is an $L^{1}$-approximation of $u_{\varepsilon}$ and bounded below, and since $u_{\varepsilon}$ converges pointwise (cf Remark 3.2), we infer that $f_{\varepsilon}$ itself has a pointwise limit. Using Lemma 3.4, we may as well assume that $\nabla f_{\varepsilon}$ converges weakly.

Lemma 3.6. Assume that $\Omega$ is the unit ball and that the initial data $\varphi_{\varepsilon}^{0}$ are radially symmetric. Then for all $T>t_{2}>t_{1}>0$ and all $1>r>0$

$$
\varepsilon^{2}\left|u_{\varepsilon}^{2} f_{\varepsilon}^{\prime}\left(t_{2}, r\right)\right|^{2} \leq \frac{C}{r^{n-1}}\left(\left.E_{\varepsilon}\left(\varphi_{\varepsilon}\right)\right|_{t_{2}} ^{t_{1}}+\varepsilon^{3} \int_{\Omega}\left|\partial_{t} \varphi_{\varepsilon}\left(t_{1}, x\right)\right|^{2} d x\right)^{\frac{1}{2}} .
$$

Proof. We integrate (E3) over the set $\{r<|x|<s\}$ and then take the mean value over $s \in(r, r+\delta)$ :

$$
\begin{aligned}
\left|\omega_{n} r^{n-1} u_{\varepsilon}^{2} f_{\varepsilon}^{\prime}\left(t_{2}, r\right)\right|^{2}=\left|\int_{|x|=r}\left(u_{\varepsilon}^{2} \nabla f_{\varepsilon}\right)\left(t_{2}, x\right) \cdot \frac{x}{|x|} d \mathcal{H}^{n-1}(x)\right|^{2} \\
\leq\left(\frac{1}{\delta} \int_{r}^{r+\delta} \int_{|x|=s}\left(u_{\varepsilon}^{2}\left|\nabla f_{\varepsilon}\right|\right)\left(t_{2}, x\right) d \mathcal{H}^{n-1}(x) d s\right. \\
\left.\quad+\frac{1}{\delta} \int_{r}^{r+\delta} \int_{r<|x|<s}\left(u_{\varepsilon}^{2}\left|\partial_{t} f_{\varepsilon}\right|\right)\left(t_{2}, x\right) d x d s\right)^{2} \\
\leq C r^{n-1}\left(\frac{1}{\delta} \int_{\Omega}\left(u_{\varepsilon}^{4}\left|\nabla f_{\varepsilon}\right|^{2}\right)\left(t_{2}, x\right) d x+\delta \int_{\Omega}\left(u_{\varepsilon}^{4}\left|\partial_{t} f_{\varepsilon}\right|^{2}\right)\left(t_{2}, x\right) d x\right)
\end{aligned}
$$

But $u_{\varepsilon}$ is uniformly bounded in $L^{\infty}\left(\Omega_{T}\right)$, so that (due to the energy estimate) the first term is uniformly bounded in time by $\frac{C}{\delta \varepsilon}$, and by Lemma 3.3

$$
\varepsilon \int_{\Omega}\left(u_{\varepsilon}^{4}\left|\partial_{t} f_{\varepsilon}\right|^{2}\right)\left(t_{2}, x\right) d x \leq\left.\frac{C}{\varepsilon^{2}} E_{\varepsilon}\left(\varphi_{\varepsilon}\right)\right|_{t_{2}} ^{t_{1}}+\varepsilon \int_{\Omega}\left|\partial_{t} \varphi_{\varepsilon}\left(t_{1}, x\right)\right|^{2} d x .
$$

Thus choosing $\frac{1}{\delta^{2}}=\left.\frac{C}{\varepsilon^{2}} E_{\varepsilon}\left(\varphi_{\varepsilon}\right)\right|_{t_{2}} ^{t_{1}}+\varepsilon \int_{\Omega}\left|\partial_{t} \varphi_{\varepsilon}\right|^{2}\left(t_{1}, x\right) d x$ proves the lemma, since $u_{\varepsilon}$ is uniformly bounded below.

Remark 3.7. As a by-product of the above proof and Lemma 3.4 we obtain

$$
\int_{0}^{T}\left|u_{\varepsilon}^{2} f_{\varepsilon}^{\prime}\right|^{2}(t, r) d t \leq \frac{C}{r^{n-1}} \frac{1}{\sqrt{\varepsilon}}
$$

by choosing $\delta=\sqrt{\varepsilon}$.

Lemma 3.8. Assume that $\Omega$ is a ball and that the initial data are radially symmetric. Then

$$
\varepsilon \int_{0}^{T} \int_{0}^{1}\left|\nabla \varphi_{\varepsilon}\right|^{2} r^{n-3} d r d t+\frac{1}{\varepsilon} \int_{0}^{T} \int_{0}^{1} W\left(\left|\varphi_{\varepsilon}\right|\right) r^{n-3} d r d t \leq C
$$


Proof. The differential equation (E1) written in the radial variable reads

$$
\varepsilon \partial_{t} \varphi_{\varepsilon}-\varepsilon \varphi_{\varepsilon}^{\prime \prime}-\varepsilon \frac{n-1}{r} \varphi_{\varepsilon}^{\prime}+\frac{1}{\varepsilon} \tilde{W}_{, \varphi}\left(\varphi_{\varepsilon}\right)=0
$$

We multiply this by $-r^{n-2} \varphi_{\varepsilon}^{\prime}$, integrate the resulting identity over $(0, s) \subset(0,1)$ and finally integrate by parts. This yields

$$
\begin{gathered}
\varepsilon \frac{n}{2} \int_{0}^{s}\left(\varphi_{\varepsilon}^{\prime}\right)^{2} r^{n-3} d r+\frac{\varepsilon}{2}\left|\varphi_{\varepsilon}^{\prime}\right|^{2} s^{n-2}+\frac{n-2}{\varepsilon} \int_{0}^{s} W\left(u_{\varepsilon}\right) r^{n-3} d r \\
=\frac{1}{\varepsilon} W\left(u_{\varepsilon}\right) s^{n-2}+\varepsilon \int_{0}^{s} \partial_{t} \varphi_{\varepsilon} \varphi_{\varepsilon}^{\prime} r^{n-2} d r .
\end{gathered}
$$

We use Hölder's inequality for the last term, and obtain

$$
\begin{gathered}
\varepsilon \frac{n-1}{2} \int_{0}^{s}\left(\varphi_{\varepsilon}^{\prime}\right)^{2} r^{n-3} d r+\frac{n-2}{\varepsilon} \int_{0}^{s} W\left(u_{\varepsilon}\right) r^{n-3} d r \\
\leq \frac{1}{\varepsilon} W\left(u_{\varepsilon}\right) s^{n-2}+\varepsilon \int_{0}^{s}\left|\partial_{t} \varphi_{\varepsilon}\right|^{2} r^{n-1} d r .
\end{gathered}
$$

Now we take the mean value of the right hand side over $s \geq \frac{1}{2}$ and bound the left hand side from below by the integral over $\left(0, \frac{1}{2}\right)$, and obtain

$$
\begin{aligned}
& \varepsilon \frac{n-1}{2} \int_{0}^{T} \int_{0}^{\frac{1}{2}}\left(\varphi_{\varepsilon}^{\prime}\right)^{2} r^{n-3} d r d t+\frac{n-2}{\varepsilon} \int_{0}^{T} \int_{0}^{\frac{1}{2}} W\left(u_{\varepsilon}\right) r^{n-3} d r d t \\
& \quad \leq 2 \int_{0}^{T} \int_{\frac{1}{2}}^{1} \frac{1}{\varepsilon} W\left(u_{\varepsilon}\right) s^{n-2} d s d t+\varepsilon \int_{0}^{T} \int_{0}^{1}\left|\partial_{t} \varphi_{\varepsilon}\right|^{2} r^{n-1} d r d t \leq C .
\end{aligned}
$$

This implies the assertion of the lemma.

\section{Definition of the $\varepsilon$-Interfaces AND AN APPROXIMATION OF THE SOLUTION}

In this and all the following sections we will assume that the domain $\Omega$ is the unit ball and all functions are radially symmetric. We will denote by ' the derivative with respect to the radial variable.

The energy $E_{\varepsilon}\left(\varphi_{\varepsilon}\right)$ is a uniformly bounded and decreasing function of time. Therefore a subsequence $E_{\varepsilon}\left(\varphi_{\varepsilon}\right)$ converges pointwise to a limit $E_{0}$, which is also decreasing. So for any $\eta>0$, the set

$$
N(\eta):=\left\{t \in(0, T) \mid \operatorname{ess} \inf _{s<t} E_{0}(s)-\operatorname{ess} \sup _{s>t} E_{0}(s)>\eta\right\},
$$

which is the set of points where $E_{0}$ has a jump of at least height $\eta$, is finite.

Let $c_{\text {crit }}$ be a small positive number and $a<\lambda_{\text {crit }}^{-}<d<\lambda_{\text {crit }}^{+}<b$ two level values such that in particular

$$
W(\lambda)>c_{\text {crit }} \quad \text { for all } \lambda_{\text {crit }}^{-}<\lambda<\lambda_{\text {crit }}^{+},
$$

and such that $c_{c r i t}$ and $d-\lambda_{\text {crit }}^{-}$and $\lambda_{\text {crit }}^{+}-d$ are as close to zero as required in Proposition 4.9 and Proposition 4.10. (We recall that $d$ is the maximum point of $W$ in the spinodal region and was introduced in hypothesis (W1).)

Proposition 4.1. For any $\frac{1}{2}>R_{0}>0$ there is an $\eta\left(R_{0}\right)$ such that for any $0<$ $t_{0} \notin N\left(\eta\left(R_{0}\right)\right)$, there exist $T_{0}=T_{0}\left(t_{0}, R_{0}\right)$ and $\varepsilon_{0}=\varepsilon_{0}\left(t_{0}, R_{0}\right)$ such that

$$
\sup _{t_{0}-T_{0}<t<t_{0}+T_{0}}\left\|\varepsilon^{2}\left|u_{\varepsilon}^{\prime}\right|^{2}-2 W\left(u_{\varepsilon}\right)\right\|_{L^{\infty}\left(R_{0}, 1\right)}(t) \leq c_{\text {crit }} \quad \text { for all } \varepsilon \leq \varepsilon_{0} .
$$


Proof. If $0<t_{0} \notin N(\eta)$, then there exists $T_{0}=T_{0}\left(t_{0}, \eta\right)$ such that $\left.E_{\varepsilon}\left(\varphi_{\varepsilon}\right)\right|_{t_{0}-T_{0}} ^{t_{0}+T_{0}} \leq \eta$ for all $\varepsilon \leq \varepsilon_{0}=\varepsilon_{0}\left(t_{0}, \eta\right)$. To see this, define $T_{\varepsilon}$ by $\left.E_{\varepsilon}\left(\varphi_{\varepsilon}\right)\right|_{\left(t_{0}-T_{\varepsilon}\right)+} ^{t_{0} T_{\varepsilon}}=\eta$ or, if there is no such $T_{\varepsilon}$, by $+\infty$. Then by the definition of $N(\eta)$ we find that $T_{\varepsilon}$ is bounded below. Define $T_{0}$ to be this lower bound.

Let $t \in\left(t_{0}-\frac{T_{0}}{2}, t_{0}+T_{0}\right)$.

We now use the differential equation (E1), written in the radial variable:

$$
\varepsilon \partial_{t} \varphi_{\varepsilon}-\varepsilon \varphi_{\varepsilon}^{\prime \prime}-\varepsilon \frac{n-1}{r} \varphi_{\varepsilon}^{\prime}+\frac{1}{\varepsilon} \tilde{W}_{, \varphi}\left(\varphi_{\varepsilon}\right)=0
$$

Choose $r>R_{0}$ arbitrarily. We multiply the above differential equation by $\varphi_{\varepsilon}^{\prime}$, and for any $s \in\left(R_{0}, 1\right)$ we integrate over either $(r, s)$ or $(s, r)$. Next take absolute values and integrate over $s \in\left(R_{0}, 1\right)$. Then we use Hölder's inequality and the bound on the energy to arrive at

$$
\begin{aligned}
& \left.\left|\varepsilon^{2}\right| \varphi_{\varepsilon}^{\prime}\right|^{2}-2 \tilde{W}\left(\varphi_{\varepsilon}\right) \mid(t, r) \\
\leq & C\left(R_{0}\right)\left(\varepsilon \int_{\Omega}\left(\frac{\varepsilon}{2}\left|\nabla \varphi_{\varepsilon}\right|^{2}+\frac{1}{\varepsilon} W\left(\left|\varphi_{\varepsilon}\right|\right)\right) d x+\left(\varepsilon^{4} \int_{\Omega}\left|\partial_{t} \varphi_{\varepsilon}\right|^{2} d x \int_{\Omega}\left|\nabla \varphi_{\varepsilon}\right|^{2} d x\right)^{\frac{1}{2}}\right. \\
& \left.+\varepsilon^{2} \int_{\Omega}\left|\nabla \varphi_{\varepsilon}\right|^{2} d x\right)(t) \\
\leq & C\left(R_{0}\right)\left(\varepsilon+\left(\varepsilon^{3} \int_{\Omega}\left|\partial_{t} \varphi_{\varepsilon}\right|^{2}(t, x) d x\right)^{\frac{1}{2}}\right) .
\end{aligned}
$$

As a consequence

$$
\begin{aligned}
& \left.\left|\varepsilon^{2}\right| u_{\varepsilon}^{\prime}\right|^{2}-2 W\left(u_{\varepsilon}\right) \mid(t, r) \\
& \quad \leq C\left(R_{0}\right)\left(\varepsilon+\left(\varepsilon^{3} \int_{\Omega}\left|\partial_{t} \varphi_{\varepsilon}\right|^{2}(t, x)\right)^{\frac{1}{2}}\right) d x+\varepsilon^{2}\left(u_{\varepsilon}^{2}\left|f_{\varepsilon}^{\prime}\right|^{2}\right)(t, r) .
\end{aligned}
$$

We use Lemma 3.3 with $t_{2}=t>t_{1}$ to estimate the $\partial_{t} \varphi_{\varepsilon}$-term and Lemma 3.6 to estimate the $f_{\varepsilon}^{\prime}$-term. We find that

$$
\begin{aligned}
& \left.\left|\varepsilon^{2}\right| u_{\varepsilon}^{\prime}\right|^{2}-2 W\left(u_{\varepsilon}\right) \mid(t, r) \\
& \quad \leq C\left(R_{0}\right)\left(\varepsilon+\left(\left.E_{\varepsilon}\left(\varphi_{\varepsilon}\right)\right|_{t} ^{t_{1}}\right)^{\frac{1}{2}}+\left(\varepsilon^{3} \int_{\Omega}\left|\partial_{t} \varphi_{\varepsilon}\right|^{2}\left(t_{1}, x\right) d x\right)^{\frac{1}{2}}\right)
\end{aligned}
$$

for all $r>R_{0}$ and all $t>t_{1}$.

Since $t>t_{0}-\frac{T_{0}}{2}$ we may choose $t_{1} \in\left(t_{0}-T_{0}, t_{0}-\frac{T_{0}}{2}\right)$, and by the definition of $T_{0}$ we obtain

$$
\left.\left|\varepsilon^{2}\right| u_{\varepsilon}^{\prime}\right|^{2}-2 W\left(u_{\varepsilon}\right) \mid(t, r) \leq C\left(R_{0}\right)\left(\varepsilon+\eta^{\frac{1}{2}}+\left(\varepsilon^{3} \int_{\Omega}\left|\partial_{t} \varphi_{\varepsilon}\right|^{2}\left(t_{1}, x\right) d x\right)^{\frac{1}{2}}\right) .
$$

Taking the mean value over $t_{1} \in\left(t_{0}-T_{0}, t_{0}-\frac{T_{0}}{2}\right)$, using Hölder's inequality and the energy bound for $\partial_{t} \varphi_{\varepsilon}$ finally yields

$$
\left.\left|\varepsilon^{2}\right| u_{\varepsilon}^{\prime}\right|^{2}-2 W\left(u_{\varepsilon}\right) \mid(t, r) \leq C\left(R_{0}\right)\left(\varepsilon+\eta^{\frac{1}{2}}+\left(\frac{2}{T_{0}}\right)^{\frac{1}{2}} \varepsilon\right) .
$$

We define $\eta\left(R_{0}\right)$ by $C\left(R_{0}\right) \eta^{\frac{1}{2}}=\frac{c_{\text {crit }}}{2}$ and choose $\varepsilon_{0}\left(t_{0}, R_{0}\right)$ small enough in order to conclude. 
Remark 4.2. As a byproduct of the above proof we obtain

$$
\int_{0}^{T}\left\|\varepsilon^{2}\left|u_{\varepsilon}^{\prime}\right|^{2}-2 W\left(u_{\varepsilon}\right)\right\|_{L^{\infty}\left(R_{0}, 1\right)}(t) d t \leq C\left(R_{0}\right) \varepsilon .
$$

Definition 4.3. For any $R_{0}>0$ we redefine $N\left(R_{0}\right)$ by $N\left(\eta\left(R_{0}\right)\right)$, and we let

$$
A\left(R_{0}\right):=\bigcup_{0<t \notin N\left(R_{0}\right)}\left(t_{0}-T_{0}\left(t_{0}, R_{0}\right), t_{0}+T_{0}\left(t_{0}, R_{0}\right)\right)
$$

and note that its complement in $[0, T]$ consists of only finitely many points. We choose an increasing sequence of open sets $B=B_{m}$ that are compact in $A\left(R_{0}\right)$ and such that $A\left(R_{0}\right)=\bigcup B_{m}$.

Furthermore we define for any $R_{0}>0$ and any $\lambda \in\left(\lambda_{\text {crit }}^{-}, \lambda_{\text {crit }}^{+}\right)$the $\varepsilon$-free boundary

$$
\Gamma_{\varepsilon}\left(R_{0}, \lambda\right):=\left\{(t, r) \in(0, T) \times\left(R_{0}, 1\right] \mid u_{\varepsilon}(t, r)=\lambda\right\} .
$$

Corollary and Definition 4.4. For any $R_{0}>0$ there exists $\lambda_{\varepsilon}=\lambda_{\varepsilon}\left(R_{0}\right) \in$ $\left(\lambda_{\text {crit }}^{-}, \lambda_{\text {crit }}^{+}\right)$, such that for any $B=B_{m}$ as in Definition 4.3 and for any $\varepsilon \leq$ $\varepsilon_{0}\left(B, R_{0}\right)$ the set

$$
\Gamma_{\varepsilon}\left(R_{0}, \lambda_{\varepsilon}\right) \cap B \times\left(R_{0}, 1\right]
$$

consists of a collection of graphs

$$
r_{\varepsilon}^{i}: I_{\varepsilon}^{i} \subset B \longrightarrow\left(R_{0}, 1\right) \quad \text { for } i=1, \ldots, M_{\varepsilon},
$$

with $r_{\varepsilon}^{i}>r_{\varepsilon}^{i+1}$ and $r_{\varepsilon}^{i}=R_{0}$ on $\partial I_{\varepsilon}^{i} \cap B$. In addition, $M_{\varepsilon} \leq C\left(R_{0}\right)$ and

$$
\int_{B} \sum_{i}\left|\frac{d}{d t} r_{\varepsilon}^{i}(t)\right|^{2} d t \leq C\left(R_{0}\right) .
$$

Proof. By Proposition 4.1 all level sets with value in the region $\left(\lambda_{\text {crit }}^{-}, \lambda_{\text {crit }}^{+}\right)$are graphs. So we may define $r_{\varepsilon}^{i}(t, \lambda)$ by $u_{\varepsilon}\left(t, r_{\varepsilon}^{i}(t, \lambda)\right)=\lambda$. Using the co-area formula, the bound for $\frac{1}{\left|u_{\varepsilon}^{\prime}\right|}$ given by Proposition 4.1 and the energy bound for $\partial_{t} u_{\varepsilon}$, we can select a particular value $\lambda_{\varepsilon} \in\left(\lambda_{\text {crit }}^{-}, \lambda_{\text {crit }}^{+}\right)$so that $r_{\varepsilon}^{i}\left(t, \lambda_{\varepsilon}\right)$ is uniformly bounded in $H^{1,2}$ as follows:

$$
\begin{gathered}
\int_{B} \int_{\lambda_{\text {crit }}^{-}}^{\lambda_{\text {crit }}^{+}} \sum_{i}\left|\partial_{t} r_{\varepsilon}^{i}(t, \lambda)\right|^{2} d \lambda d t \leq \int_{B} \int_{W\left(u_{\varepsilon}\right)>c_{c r i t}} \frac{\left|\partial_{t} u_{\varepsilon}(t, r)\right|^{2}}{\left|u_{\varepsilon}^{\prime}(t, r)\right|} d r d t \\
\leq \frac{1}{\left\|u_{\varepsilon}^{\prime}\right\|_{L^{\infty}}} \int_{0}^{T} \int_{\Omega}\left|\partial_{t} u_{\varepsilon}\right|^{2} d x d t \leq C\left(R_{0}\right) \quad \text { for } \varepsilon \leq \varepsilon_{0}\left(B, R_{0}\right) .
\end{gathered}
$$

Then we define for any $R_{0}$ the set $\Lambda(B):=\left\{\lambda\left|\sum_{i} \int_{B}\right| \dot{r}_{\varepsilon}^{i}(t, \lambda) \mid d t \leq C\left(R_{0}\right)\right.$ for all $\left.\varepsilon \leq \varepsilon_{0}\left(B, R_{0}\right)\right\}$ and show that $\Lambda\left(B^{\prime}\right) \subset \Lambda(B)$ if $B \subset B^{\prime}$ and that $|\Lambda(B)| \geq c_{0}\left(R_{0}\right)$. Thus we may choose $\lambda_{\varepsilon} \in \bigcap_{B} \Lambda(B)$, and we define $r_{\varepsilon}^{i}(t):=r_{\varepsilon}^{i}\left(t, \lambda_{\varepsilon}\right)$.

Proposition 4.1 and the Neumann condition for $u_{\varepsilon}$ imply furthermore that this level set will never hit $\partial \Omega$. In addition, combining Proposition 4.1 and the bound for the energy, we find that the number of interfaces is uniformly bounded.

Definition 4.5. Let $R_{0}$ and $B=B_{m}$ be as in Definition 4.3. For $r>R_{0}$ and $t \in B$ we define

$$
z:=\frac{r}{\varepsilon}, \quad U_{\varepsilon}(t, z):=u_{\varepsilon}(t, r) \quad \text { and } \quad z_{\varepsilon}^{i}(t):=\frac{r_{\varepsilon}^{i}(t)}{\varepsilon}\left(i=1, \ldots, M_{\varepsilon}\right) .
$$


Next we define $\tau=\tau_{\varepsilon}$ to be either 0 or 1 , according to whether $u_{\varepsilon}$ is close to $a$ or $b$ on $\partial \Omega$. This is locally constant in time. In addition we define the stationary wave solution $Q: \mathbf{R} \rightarrow \mathbf{R}$ by

$$
Q^{\prime \prime}=W^{\prime}(Q), \quad Q(-\infty)=a, \quad Q(+\infty)=b, \quad Q(0)=d,
$$

and $Q_{\varepsilon}(z):=Q\left(z+\mu_{\varepsilon}\right)$, where $\mu_{\varepsilon}$ is such that $Q\left(\mu_{\varepsilon}\right)=\lambda_{\varepsilon}$. We choose a smooth, increasing function $\Xi$ with $\Xi(z)=1$ if $z>1$ and $\Xi(z)+\Xi(-z)=1$. Furthermore we define the first order approximation

$$
V_{\varepsilon}(t, z):=\sum_{i=1}^{M_{\varepsilon}} \Xi_{\varepsilon}^{i}(t, z) V_{\varepsilon}^{i}(t, z):=\sum_{i=1}^{M_{\varepsilon}} \Xi_{\varepsilon}^{i}(t, z) Q_{\varepsilon}\left((-1)^{i+\tau_{\varepsilon}}\left[z-z_{\varepsilon}^{i}(t)\right]\right),
$$

where $\Xi_{\varepsilon}^{i}$ is a partition of unity, given by

$$
\Xi_{\varepsilon}^{i}(t, z):= \begin{cases}\Xi\left(z-\frac{z_{\varepsilon}^{i}(t)+z_{\varepsilon}^{i+1}(t)}{2}\right), & \text { if } z \leq z_{\varepsilon}^{i}(t), \\ \Xi\left(\frac{z_{\varepsilon}^{i}(t)+z_{\varepsilon}^{i-1}(t)}{2}-z\right), & \text { if } z \geq z_{\varepsilon}^{i}(t),\end{cases}
$$

for $i=2, \ldots, M_{\varepsilon}-1$, and by

$$
\begin{gathered}
\Xi_{\varepsilon}^{1}(t, z):= \begin{cases}\Xi\left(z-\frac{z_{\varepsilon}^{1}(t)+z_{\varepsilon}^{2}(t)}{2}\right), & \text { if } z \leq z_{\varepsilon}^{1}(t), \\
1, & \text { if } z \geq z_{\varepsilon}^{1}(t),\end{cases} \\
\Xi_{\varepsilon}^{M_{\varepsilon}}(t, z):= \begin{cases}1, & \text { if } z \leq z_{\varepsilon}^{M_{\varepsilon}}(t), \\
\Xi\left(\frac{z_{\varepsilon}^{M_{\varepsilon}}(t)+z_{\varepsilon}^{M_{\varepsilon}-1}(t)}{2}-z\right), & \text { if } z \geq z_{\varepsilon}^{M_{\varepsilon}}(t) .\end{cases}
\end{gathered}
$$

Lemma 4.6 below implies that this is well defined.

Finally we define the difference

$$
Y_{\varepsilon}:=U_{\varepsilon}-V_{\varepsilon}
$$

In the remainder of this section we will show that $Y_{\varepsilon}$ is small.

Lemma 4.6. Let $R_{0}$ and $B=B_{m}$ be as in Definition 4.3. Then there exists a constant $M\left(c_{\text {crit }}\right)$, that converges to zero as $c_{\text {crit }}$ goes to zero, such that for all $\varepsilon \leq \varepsilon_{0}\left(B, R_{0}\right)$

$$
\sup _{B}\left\|Y_{\varepsilon}\right\|_{L^{\infty}\left(R_{0} / \varepsilon, 1 / \varepsilon\right)} \leq M\left(c_{c r i t}\right)
$$

and

$$
\sup _{B}\left|z_{\varepsilon}^{i}-z_{\varepsilon}^{i+1}\right| \geq \frac{1}{M\left(c_{\text {crit }}\right)}
$$

for $i=1, \ldots, M_{\varepsilon}-1$. In addition, for almost all $t \in B$

$$
\left|z_{\varepsilon}^{i}-z_{\varepsilon}^{i+1}\right| \longrightarrow \infty
$$

Proof. Proposition 4.1 implies a differential equation for $U_{\varepsilon}$ :

$$
\left|U_{\varepsilon}^{\prime}\right|=\sqrt{2 W\left(U_{\varepsilon}\right)}+K_{\varepsilon}
$$

where $\left\|K_{\varepsilon}\right\|_{L^{\infty}} \leq c_{\text {crit }}$ and $\int_{B}\left\|K_{\varepsilon}\right\|_{L^{\infty}} \leq C\left(R_{0}\right) \varepsilon$. Lemma 4.6 follows by direct integration of this equation. 
Remark and Definition 4.7. The difference $Y_{\varepsilon}$ satisfies the differential equation

$$
-Y_{\varepsilon}^{\prime \prime}+W^{\prime \prime}\left(V_{\varepsilon}\right) Y_{\varepsilon}=G_{\varepsilon}-H_{\varepsilon}-\int_{V_{\varepsilon}}^{V_{\varepsilon}+Y_{\varepsilon}} W^{\prime \prime \prime}(\lambda)\left(V_{\varepsilon}+Y_{\varepsilon}-\lambda\right) d \lambda
$$

where

$$
G_{\varepsilon}(t, z):=\left[-\varepsilon^{2} \partial_{t} u_{\varepsilon}+\varepsilon^{2} \frac{n-1}{r} u_{\varepsilon}^{\prime}-\varepsilon^{2} u_{\varepsilon}\left|f_{\varepsilon}^{\prime}\right|^{2}\right](t, \varepsilon z)
$$

and

$$
\begin{aligned}
H_{\varepsilon}:=\sum_{i}( & \left(\Xi_{\varepsilon}^{i}\right)^{\prime \prime} V_{\varepsilon}^{i}+2\left(\Xi_{\varepsilon}^{i}\right)^{\prime}\left(V_{\varepsilon}^{i}\right)^{\prime} \\
& \left.+\Xi_{\varepsilon}^{i} W^{\prime \prime}\left(V_{\varepsilon}^{i}\right)\left(V_{\varepsilon}-V_{\varepsilon}^{i}\right)+\Xi_{\varepsilon}^{i} \int_{V_{\varepsilon}^{i}}^{V_{\varepsilon}} W^{\prime \prime \prime}(\lambda)\left(V_{\varepsilon}-\lambda\right) d \lambda\right) .
\end{aligned}
$$

In addition, $Y_{\varepsilon}\left(t, z_{\varepsilon}^{i}(t)\right)=0$ for $i=1, \ldots, M_{\varepsilon}$.

Lemma 4.8. Let $R_{0}$ and $B=B_{m}$ be as in Definition 4.3. Then for $\varepsilon \leq \varepsilon_{0}\left(B, R_{0}\right)$

$$
\int_{B} \int_{R_{0} / \varepsilon}^{1 / \varepsilon}\left|G_{\varepsilon}\right|^{2} d z d t \leq C\left(R_{0}\right) \varepsilon^{3 / 2}
$$

and for $i=2, \ldots, M_{\varepsilon}$

$$
\begin{gathered}
\int_{B} \int_{z_{\varepsilon}^{i}(t)}^{z_{\varepsilon}^{i-1}(t)}\left|H_{\varepsilon}\right|^{2} d z d t \leq C\left(R_{0}\right) \int_{B} \frac{\varepsilon^{2}}{\left(r_{\varepsilon}^{i-1}-r_{\varepsilon}^{i}\right)^{2}(t)} d t \\
\int_{B} \int_{z_{\varepsilon}^{i}(t)}^{z_{\varepsilon}^{i-1}(t)} \int_{V_{\varepsilon}}^{V_{\varepsilon}+Y_{\varepsilon}}\left|W^{\prime \prime \prime}(\lambda)\left(V_{\varepsilon}+Y_{\varepsilon}-\lambda\right)\right| d \lambda\left|Y_{\varepsilon}\right| d z d t \\
\leq C\left(R_{0}\right) M\left(c_{c r i t}\right) \int_{B} \int_{z_{\varepsilon}^{i}(t)}^{z_{\varepsilon}^{i-1}(t)}\left|Y_{\varepsilon}\right|^{2} d z d t
\end{gathered}
$$

and the same is true for $i=1$ and $i=M_{\varepsilon}+1$, when formally setting $r_{\varepsilon}^{0}=1$ and $r_{\varepsilon}^{M_{\varepsilon}+1}=R_{0}$, and hence $z_{\varepsilon}^{0}=\frac{1}{\varepsilon}$ and $z_{\varepsilon}^{M_{\varepsilon}+1}=\frac{R_{0}}{\varepsilon}$.

Proof. The first inequality follows from the bounds derived in Remarks 3.1 and 3.7.

The second inequality follows directly from the precise knowledge of the stationary wave solution $Q$ and of the partition of unity $\Xi$. Indeed the sum is only taken over two integers at a time, and in the support of $\left(\Xi_{\varepsilon}^{i}\right)^{\prime} \cap\left(z_{\varepsilon}^{i}, z_{\varepsilon}^{i-1}\right)$ the difference $\left|V_{\varepsilon}^{i}-V_{\varepsilon}^{i-1}\right|$ is bounded by $\frac{C}{z_{\varepsilon}^{i-1}-z_{\varepsilon}^{i}}$.

The last inequality is straightforward, since $\left\|Y_{\varepsilon}\right\|_{L^{\infty}\left(B \times\left(R_{0} / \varepsilon, 1 / \varepsilon\right)\right)} \leq M\left(c_{\text {crit }}\right)$ by Lemma 4.6.

Proposition 4.9. Let $R_{0}$ and $B=B_{m}$ be as in Definition 4.3. If $c_{\text {crit }}$ is suffciently small and $\lambda_{\text {crit }}^{-}$and $\lambda_{\text {crit }}^{+}$are sufficiently close to $d$, then there are positive constants $\eta_{1}$ and $\eta_{2}$ such that for any $i=2, \ldots, M_{\varepsilon}$

$$
\int_{z_{\varepsilon}^{i}(t)}^{z_{\varepsilon}^{i-1}(t)}\left[-Y_{\varepsilon}^{\prime \prime}+W^{\prime \prime}\left(V_{\varepsilon}\right) Y_{\varepsilon}\right] Y_{\varepsilon} d z \geq \eta_{1} \int_{z_{\varepsilon}^{i}(t)}^{z_{\varepsilon}^{i-1}(t)}\left|Y_{\varepsilon}^{\prime}\right|^{2} d z+\eta_{2} \int_{z_{\varepsilon}^{i}(t)}^{z_{\varepsilon}^{i-1}(t)} Y_{\varepsilon}^{2} d z
$$


and

$$
\int_{z_{\varepsilon}^{1}(t)}^{1 / \varepsilon}\left[-Y_{\varepsilon}^{\prime \prime}+W^{\prime \prime}\left(V_{\varepsilon}\right) Y_{\varepsilon}\right] Y_{\varepsilon} d z \geq \eta_{1} \int_{z_{\varepsilon}^{1}(t)}^{1 / \varepsilon}\left|Y_{\varepsilon}^{\prime}\right|^{2} d z+\eta_{2} \int_{z_{\varepsilon}^{1}(t)}^{1 / \varepsilon} Y_{\varepsilon}^{2} d z-\left(Y_{\varepsilon} Y_{\varepsilon}^{\prime}\right)\left(\frac{1}{\varepsilon}\right)
$$

and

$$
\begin{aligned}
\int_{R_{0} / \varepsilon}^{z_{\varepsilon}^{M_{\varepsilon}}(t)}\left[-Y_{\varepsilon}^{\prime \prime}+W^{\prime \prime}\left(V_{\varepsilon}\right) Y_{\varepsilon}\right] Y_{\varepsilon} Z d z & \\
& \geq \eta_{1} \int_{R_{0} / \varepsilon}^{z_{\varepsilon}^{M_{\varepsilon}}(t)}\left|Y_{\varepsilon}^{\prime}\right|^{2} Z d z+\eta_{2} \int_{R_{0} / \varepsilon}^{z_{\varepsilon}^{M_{\varepsilon}}(t)} Y_{\varepsilon}^{2} Z d z-\frac{1}{2} \int_{R_{0} / \varepsilon}^{z_{\varepsilon}^{M_{\varepsilon}}(t)} Y_{\varepsilon}^{2} Z^{\prime \prime} d z
\end{aligned}
$$

for any smooth, positive test function $Z$ with $Z\left(\frac{R_{0}}{\varepsilon}\right)=Z^{\prime}\left(\frac{R_{0}}{\varepsilon}\right)=0$.

Proof. This proposition follows from an idea of Berger and Fraenkel (1970). We sketch the proof for the first inequality: Define $I_{\varepsilon}:=\left\{z \mid W^{\prime \prime}\left(V_{\varepsilon}\right) \leq \eta_{2}\right\}$. If $c_{\text {crit }}$, $d-\lambda_{\text {crit }}^{-}$and $\lambda_{\text {crit }}^{+}-d$ as well as $\eta_{2}$ are sufficiently small, then by Definition 4.5 and Lemma 4.6

$$
I_{\varepsilon}=\bigcup_{i}\left(z_{\varepsilon}^{i}(t), z_{\varepsilon}^{i}(t)+l^{+}\right) \cup\left(z_{\varepsilon}^{i-1}(t)-l^{-}, z_{\varepsilon}^{i-1}(t)\right)+o(1),
$$

where $\left(-l^{-}, l^{+}\right):=\left\{z \mid W^{\prime \prime}(Q) \leq 0\right\}$. The $o(1)$-term converges to zero with $c_{\text {crit }}$, $d-\lambda_{\text {crit }}^{-}$and $\lambda_{\text {crit }}^{+}-d$ and $\eta_{2}$ independently of $\varepsilon$. Consequently

$$
\begin{gathered}
\int\left(\left|Y_{\varepsilon}^{\prime}\right|^{2}+W^{\prime \prime}\left(V_{\varepsilon}\right) Y_{\varepsilon}^{2}\right) d z \geq \int\left|Y_{\varepsilon}^{\prime}\right|^{2} d z-\left(\eta_{2}+\left|\min W^{\prime \prime}\right|\right) \int_{I} Y_{\varepsilon}^{2} d z+\eta_{2} \int Y_{\varepsilon}^{2} d z \\
\geq\left(1-\left(\frac{1}{2} \max ^{2}\left(l^{-}, l^{+}\right)+o(1)\right)\left(\eta_{2}+\left|\min W^{\prime \prime}\right|\right)\right) \int\left|Y_{\varepsilon}^{\prime}\right|^{2} d z+\eta_{2} \int Y_{\varepsilon}^{2} d z,
\end{gathered}
$$

where we have used the fact that by construction $Y_{\varepsilon}\left(t, z_{\varepsilon}^{i-1}(t)\right)=Y_{\varepsilon}\left(t, z_{\varepsilon}^{i}(t)\right)=0$. It turns out that $1-\frac{1}{2} \max ^{2}\left(l^{-}, l^{+}\right)\left|\min W^{\prime \prime}\right|$ is positive, and thus the proposition follows. For more details, we refer to the appendix of [BSt].

Proposition 4.10. Let $R_{0}$ and $B=B_{m}$ be as in Definition 4.3 and assume that $c_{\text {crit }}$ is sufficiently small and $\lambda_{\text {crit }}^{-}$and $\lambda_{\text {crit }}^{+}$are sufficiently close to $d$. Then for $i=2, \ldots, M_{\varepsilon}$ and any $B^{\prime} \subset B$

$$
\int_{B^{\prime}}\left\|Y_{\varepsilon}(t, \cdot)\right\|_{H^{1,2}\left(z_{\varepsilon}^{i}(t), z_{\varepsilon}^{i-1}(t)\right)}^{2} d t \leq C\left(R_{0}\right)\left(\int_{B^{\prime}} \frac{\varepsilon^{2}}{\left|r_{\varepsilon}^{i-1}(t)-r_{\varepsilon}^{i}(t)\right|^{2}} d t+\varepsilon^{3 / 2}\right),
$$

and

$$
\int_{B^{\prime}}\left\|Y_{\varepsilon}(t, \cdot)\right\|_{H^{1, \infty}\left(z_{\varepsilon}^{i}(t), z_{\varepsilon}^{i-1}(t)\right)}^{2} d t \leq C\left(R_{0}\right)\left(\int_{B^{\prime}} \frac{\varepsilon^{2}}{\left|r_{\varepsilon}^{i-1}(t)-r_{\varepsilon}^{i}(t)\right|^{2}} d t+\varepsilon^{3 / 2}\right) .
$$

The same is true, if a) $\left(z_{\varepsilon}^{i}(t), z_{\varepsilon}^{i-1}(t)\right)$ is replaced by $\left(z_{\varepsilon}^{1}(t), 1 / \varepsilon\right)$ and the right hand side by $C\left(R_{0}, \delta\right) \varepsilon^{\frac{3}{2}}$ and $B^{\prime}$ is any set where $\frac{1}{\varepsilon}-z_{\varepsilon}^{1}(t) \geq \frac{\delta}{\varepsilon}$, or $\left.b\right)\left(z_{\varepsilon}^{i}(t), z_{\varepsilon}^{i-1}(t)\right)$ is replaced by $\left(\frac{R_{0}+\delta}{\varepsilon}, z_{\varepsilon}^{M_{\varepsilon}}(t)\right)$ and the right hand side by $C\left(R_{0}, \delta\right) \varepsilon^{\frac{3}{2}}$ and $B^{\prime}$ is any set where $z_{\varepsilon}^{M_{\varepsilon}}(t)-\frac{R_{0}+2 \delta}{\varepsilon} \geq 0$.

Proof. We multiply the differential equation in Remark 4.7 by $Y_{\varepsilon}$ and integrate over $\left(z_{\varepsilon}^{i}(t), z_{\varepsilon}^{i-1}(t)\right)$ or $\left(z_{\varepsilon}^{1}(t), \frac{1}{\varepsilon}\right)$ or $\left(\frac{R_{0}+\delta}{\varepsilon}, z_{\varepsilon}^{M_{\varepsilon}}(t)\right)$, resp. In the first case we use the first estimate of Proposition 4.9 to obtain the lower bound of the left hand side. Hölder's 
inequality and Lemma 4.8 imply the bound for the right hand side. In the two other cases we apply the second or third inequality of Proposition 4.9, resp. In the second case the extra boundary term is exponentially small. In the third case the extra term (we choose $Z$ as a smooth cut-off function with supp $Z^{\prime} \subset\left(\frac{R_{0}}{\varepsilon}, \frac{R_{0}+\delta}{\varepsilon}\right)$ ) is bounded by $C \frac{\varepsilon^{2}}{\delta^{2}} \int_{B^{\prime}} \int_{R_{0} / \varepsilon}^{\left(R_{0}+\delta\right) / \varepsilon}\left|Y_{\varepsilon}\right|^{2} d z d t$. But in the set of integration $V_{\varepsilon}$ is exponentially close to $a$ or $b$, which in return implies that $\left|Y_{\varepsilon}\right|=\left|U_{\varepsilon}-V_{\varepsilon}\right| \leq C \sqrt{W\left(U_{\varepsilon}\right)}$. This implies that the extra term is of order $\varepsilon^{2}$.

This proves the first part of this proposition.

Since $R_{0}$ is positive the problem is one dimensional, and the $H^{1, \infty}{ }_{-}$norm can be controlled by the $H^{2,2}$-norm. But this norm can be calculated using the equation and the estimates already obtained in this proposition.

\section{Passage to the limit in equation (E2)}

In this section we derive the limit equation of (E2). First we define the limit interface.

Definition 5.1. We choose a sequence $R_{0} \rightarrow 0$. For any $R_{0}>0$ in this sequence we define the limit interface $\Gamma\left(R_{0}\right)$ as follows.

Let $A\left(R_{0}\right)$ be as in Definition 4.3. Then by Corollary 4.4, we know that for a subsequence $\varepsilon \rightarrow 0$ there exist limits

$$
\bar{r}^{i}: I^{i} \subset A\left(R_{0}\right) \longrightarrow\left(R_{0}, 1\right) \quad \text { for } i=1, \ldots, M_{0},
$$

such that $r_{\varepsilon}^{i} \longrightarrow \bar{r}^{i}$ strongly in $C_{l o c}^{0}\left(A\left(R_{0}\right)\right)$ and $\frac{d}{d t} r_{\varepsilon}^{i} \longrightarrow \frac{d}{d t} \bar{r}^{i}$ weakly in $L_{l o c}^{2}\left(A\left(R_{0}\right)\right)$ for $i=1, \ldots, M_{0}$. In addition, $\bar{r}^{i} \geq \bar{r}^{i+1}$ and $\bar{r}^{i}=R_{0}$ on $\partial I^{i} \cap A\left(R_{0}\right)$. So we set

$$
\Gamma\left(R_{0}\right):=\left\{(t, r) \in A\left(R_{0}\right) \times\left(R_{0}, 1\right) \mid r=\bar{r}^{i}(t) \text { for some } i \in\left\{1, \ldots, M_{0}\right\}\right\} .
$$

(We note that by a diagonal argument the above subsequence can be chosen independently of $R_{0}$.) Now let $\left(t_{0}, r_{0}\right) \in \Gamma\left(R_{0}\right)$ be arbitrary. Then there exists a minimal $k$ such that $r_{0}=\bar{r}^{k}\left(t_{0}\right)$. We define the multiplicity $m_{0}\left(t_{0}, r_{0}\right)$ to be the number of $\varepsilon$-interfaces $r_{\varepsilon}^{i}\left(t_{0}\right)$, which converge to $r_{0}$. Because the $\varepsilon$-interfaces are locally uniformly bounded in $H^{1,2}$, there exists a neighbourhood $\left(t_{1}, t_{2}\right)$ of $t_{0}$, such that for $i=k, \ldots, k+m_{0}-1$,

$$
r_{\varepsilon}^{i} \rightarrow \bar{r}^{i} \quad \text { uniformly in }\left(t_{1}, t_{2}\right) \text { and weakly in } H^{1,2}\left(t_{1}, t_{2}\right)
$$

and for some $\delta>0$ and $\varepsilon \leq \varepsilon_{0}\left(t_{0}, r_{0}\right)$

$$
r_{\varepsilon}^{k}-r_{\varepsilon}^{k+1} \geq \delta \quad \text { and } \quad r_{\varepsilon}^{k+m_{0}}-r_{\varepsilon}^{k+m_{0}-1} \geq \delta \quad \text { uniformly in }\left(t_{1}, t_{2}\right) .
$$

If $m_{0}\left(t_{0}, r_{0}\right)$ is odd, we define the normal $\nu\left(t_{0}, r_{0}\right)$ by $\operatorname{sgn} u_{\varepsilon}^{\prime}\left(t_{0}, r_{\varepsilon}^{k}\left(t_{0}\right)\right)$, which for $\varepsilon \leq \varepsilon_{0}\left(t_{0}, r_{0}\right)$ does not depend on $\varepsilon$ as a consequence of Proposition 4.1 and since $u_{\varepsilon}$ has a limit in $L^{1}$ (cf. Remark 3.2). If $m_{0}\left(t_{0}, r_{0}\right)$ is even, we define $\nu\left(t_{0}, r_{0}\right):=0$. We note that $\nu$ gives the direction of the jump in the whole neighbourhood of $\left(t_{1}, t_{2}\right)$.

Proposition 5.2. Let $\left(t_{0}, r_{0}\right) \in \Gamma\left(R_{0}\right)$ be an interfacial point, and let $k, t_{1}$ and $t_{2}$ as well as $m_{0}\left(t_{0}, r_{0}\right)$ be as in Definition 5.1. Then

$$
\sum_{i=k}^{k+m_{0}-1}\left(\frac{d}{d t} \bar{r}^{i}+\frac{n-1}{\bar{r}^{i}}\right)=0 \quad \text { almost everywhere in }\left(t_{1}, t_{2}\right) .
$$


Proof. We write the differential equation (E2) in the rescaled variable $z$, multiply by $U_{\varepsilon}^{\prime}$ and integrate. Using the approximation of Section 4 , we can pass to the limit in all terms. For the $f_{\varepsilon}$-term, we use the fact that $\nabla f_{\varepsilon}$ is uniformly bounded in $L^{2}$. For more details we refer to the Appendix.

Corollary 5.3. The graphs $\bar{r}^{i}: I^{i} \subset A\left(R_{0}\right) \longrightarrow\left(R_{0}, 1\right)$ with $i=1, \ldots, M_{0}$ as in Definition 5.1 move by mean curvature:

$$
\frac{d}{d t} \bar{r}^{i}+\frac{n-1}{\bar{r}^{i}}=0 \quad \text { almost everywhere in } I^{i} .
$$

For any $i=1, \ldots, M_{0}$ the multiplicity $m_{0}\left(\cdot, \bar{r}^{i}\right)$ and the normal $\nu\left(\cdot, \bar{r}^{i}\right)$ are constant along the $i$-th interface in any connected component of $A\left(R_{0}\right)$.

We call interfaces with $\nu \neq 0$ true interfaces, whereas those with $\nu=0$ are "phantom"-interfaces, since they do not separate different phases of $u_{0}$.

Proof. Consider a set of time points where a fixed collection $i=j, . ., l$ of $\varepsilon$-interfaces have the same limit: $\bar{r}^{j}=\ldots=\bar{r}^{l}$. Then almost everywhere in this set, the derivatives with respect to time of the limit interfaces $\vec{r}^{i}$ coincide and Proposition 5.2 implies the first result. Since all the interfaces move by mean curvature, they can never meet, and so the multiplicity and the normals have to be locally constant.

Lemma and Definition 5.4. Let $t_{0} \notin A\left(R_{0}\right)$. Then for all $i \in\left\{1, \ldots, M_{0}\right\}$ with $\partial I^{i} \cap A\left(R_{0}\right)=\emptyset$ and $\lim _{s \nearrow t_{0}} \nu\left(s, \bar{r}^{i}(s)\right) \neq 0$, there exists $j \in\left\{i, \ldots, M_{0}\right\}$ with $\lim _{s \backslash t_{0}} \nu\left(s, \bar{r}^{j}(s)\right) \neq 0$ such that

$$
\lim _{s<t_{0}, s \rightarrow t_{0}} \bar{r}^{i}(s)=\lim _{s>t_{0}, s \rightarrow t_{0}} \bar{r}^{j}(s),
$$

i.e. any true interface has a continuation across points $t_{0} \notin A\left(R_{0}\right)$. Therefore we may define continuous

$$
R^{j}: D^{j} \subset(0, T) \longrightarrow\left(R_{0}, 1\right) \quad \text { for } j=1, \ldots, M_{0}^{\prime},
$$

that are given through renaming

$$
R^{j}(t):=\bar{r}^{i}(t) .
$$

Here $i=i(t, j)$ is piecewise constant in $t$, the domains of definition $D^{j}$ are connected open intervals, $\nu^{j}:=\nu\left(t, R^{j}(t)\right)$ are constant for $t \in D^{j}$ and $R^{j}(t)<R^{j-1}(t)$ in the intersection of $D^{j}$ and $D^{j-1}$.

We define the collection of true interfaces

$$
\Gamma^{\prime}\left(R_{0}\right):=\left\{(t, r) \in(0, T) \times\left(R_{0}, 1\right) \mid r=R^{j}(t) \text { for some } j=1, \ldots, M_{0}^{\prime}\right\} .
$$

(We note that $\Gamma^{\prime}$ does not depend on the subsequence selected in the preceding Definitions and Lemmata, but only on the limiting order parameter $u_{0}$ selected in Remark 3.2.) Finally we obtain $\partial_{t} u_{0}^{2} \in L^{2}\left(0, T ;\left[C^{0}\left(\left(R_{0}, 1\right]\right)\right]^{*}\right)$ and for almost all $t$ the formula

$$
\left\langle\partial_{t} u_{0}^{2}(t, \cdot), \zeta\right\rangle=\sum_{j=1}^{M_{0}^{\prime}}\left(b^{2}-a^{2}\right) \frac{d}{d t} R^{j}(t)\left(R^{j}\right)^{n-1}(t) \zeta\left(R^{j}(t)\right)
$$

for any $\zeta \in C^{0}\left(\left(R_{0}, 1\right]\right)$. 
Proof. The energy estimate implies the following weak Hölder continuity of the limiting order parameter $u_{0}$ (cf. Remark 3.2):

$$
\int_{\Omega}\left|u_{0}(t, x)-u_{0}(s, x)\right| d x \leq C|t-s|^{\frac{1}{2}} .
$$

This implies that true interfaces have continuous continuations at all time points.

Theorem 5.5. For $j=1, \ldots, M_{0}^{\prime}$, let $R^{j}$ be as in Lemma 5.4. Then

$$
\frac{d}{d t} R^{j}+\frac{n-1}{R^{j}}=0 \quad \text { in } D^{j}
$$

Proof. Continuous functions that are piecewise $H^{1,2}$ are globally $H^{1,2}$, and thus this theorem follows from Corollary 5.3 and Lemma 5.4.

\section{Passage to the limit in equation (E3)}

In this section we derive the limit equation of (E3).

Remark 6.1. We want to describe a local situation that contains at most one interface. Let $\zeta$ be a smooth test function with compact support in $(0, T) \times(0,1]$. For any such $\zeta$, there exist $R_{0}>0$ and some $0 \leq T_{1}<T_{2} \leq T$ such that $\operatorname{supp} \zeta \subset$ $\left(T_{1}, T_{2}\right) \times\left(R_{0}, 1\right)$. Since $(0, T) \backslash A\left(R_{0}\right)$ is finite, we will assume without loss of generality that there is at most one $t_{0} \in(0, T) \backslash A\left(R_{0}\right) \cap\left(T_{1}, T_{2}\right)$. Moreover, we may assume without loss of generality, that $\Gamma^{\prime}\left(R_{0}\right) \cap \operatorname{supp} \zeta=\left\{(t, r) \mid r=R^{j}(t)\right\} \cap \operatorname{supp} \zeta$ for some $j=1, \ldots, M_{0}^{\prime}$, and that $\operatorname{dist}\left(\Gamma^{\prime}\left(R_{0}\right) \backslash \operatorname{graph} R^{j} ; \operatorname{supp} \zeta\right)>0$.

Lemma 6.2. Let $\zeta$ and $R_{0}, T_{1}, T_{2}$ and $t_{0}$ be as in Definition 6.1. Let $t_{1}$ and $t_{2}$ be any two points with $T_{1}<t_{1}<t_{0}<t_{2}<T_{2}$. Then there exists $\lambda_{\varepsilon} \in\left(a+\frac{b-a}{4}, b-\frac{b-a}{4}\right)$ such that

$$
\chi_{\varepsilon}(t, x):= \begin{cases}a, & \text { if } u_{\varepsilon} \leq \lambda_{\varepsilon} \\ b, & \text { if } u_{\varepsilon}>\lambda_{\varepsilon}\end{cases}
$$

is uniformly bounded in $B V\left(\Omega_{T}\right)$ and

$$
\int_{t_{1}}^{t_{2}} \int_{\Omega}\left|\left(\partial_{t}, \nabla\right) \chi_{\varepsilon}\right| d x d t \leq C\left(t_{2}-t_{1}\right)^{\frac{1}{2}}
$$

Proof. This is a consequence of the estimate

$$
\int_{t_{1}}^{t_{2}} \int_{\Omega}\left|\left(\partial_{t}, \nabla\right) g\left(u_{\varepsilon}\right)\right| d x d t \leq C\left(t_{2}-t_{1}\right)^{\frac{1}{2}}
$$

which itself follows from the energy bound (cf. Remark 3.2). We stress that $\lambda_{\varepsilon}$ may depend on $t_{1}$ and $t_{2}$, but $C$ does not.

Theorem 6.3. Let $\zeta$ be a smooth test function with support in $(0, T) \times[0,1]$. Then

$$
-\int_{0}^{T}\left\langle\partial_{t} u_{0}^{2}, f_{0} \zeta\right\rangle d t-\int_{0}^{T} \int_{\Omega} u_{0}^{2} f_{0} \partial_{t} \zeta d x d t+\int_{0}^{T} \int_{\Omega} u_{0}^{2} \nabla f_{0} \cdot \nabla \zeta d x d t=0 .
$$

Proof. First we assume that $\operatorname{supp} \zeta \subset(0, T) \times(0,1]$ and $R_{0}, T_{1}, T_{2}, t_{0}$ and $j$ are as in Remark 6.1. We multiply equation (E3) by $\zeta$ :

$$
\int_{0}^{T} \int_{\Omega} u_{\varepsilon}^{2} \partial_{t} f_{\varepsilon} \zeta d x d t-\int_{0}^{T} \int_{\Omega} \operatorname{div}\left(u_{\varepsilon}^{2} \nabla f_{\varepsilon}\right) \zeta d x d t=0
$$


We integrate the second term by parts and pass to the limit as $\varepsilon \rightarrow 0$.

Next let $t_{1}$ and $t_{2}$ be any two points with $T_{1}<t_{1}<t_{0}<t_{2}<T_{2}$. For the first term we split the integral over $\left(T_{1}, T_{2}\right)$ into three parts: $\left(T_{1}, t_{1}\right) \cup\left(t_{1}, t_{2}\right) \cup\left(t_{2}, T_{2}\right)$.

We start with the interior integral over $\left(t_{1}, t_{2}\right)$. We write $u_{\varepsilon}^{2}$ as $\chi_{\varepsilon}^{2}+\left(u_{\varepsilon}^{2}-\chi_{\varepsilon}^{2}\right)$, where $\chi_{\varepsilon}$ is as in Lemma 6.2. Since $\left|u_{\varepsilon}^{2}-\chi_{\varepsilon}^{2}\right| \leq C \sqrt{W\left(u_{\varepsilon}\right)}$, the energy bound implies that the term with $\left(u_{\varepsilon}^{2}-\chi_{\varepsilon}^{2}\right)$ is uniformly bounded by $C\left(t_{1}-t_{2}\right)^{\frac{1}{2}}$. We integrate the $\chi_{\varepsilon}^{2}$-term by parts:

$$
\begin{aligned}
& \int_{t_{1}}^{t_{2}} \int_{\Omega} \chi_{\varepsilon}^{2} \partial_{t} f_{\varepsilon} \zeta d x d t \\
= & -\int_{t_{1}}^{t_{2}} \int_{\Omega} \partial_{t} \chi_{\varepsilon}^{2} f_{\varepsilon} \zeta d x d t-\int_{t_{1}}^{t_{2}} \int_{\Omega} \chi_{\varepsilon}^{2} f_{\varepsilon} \partial_{t} \zeta d x d t+\left.\int_{\Omega} \chi_{\varepsilon}^{2} f_{\varepsilon} \zeta d x\right|_{t_{1}} ^{t_{2}} .
\end{aligned}
$$

By Lemma 6.2 the first term is bounded by $C\left(t_{2}-t_{1}\right)^{\frac{1}{2}}$. The second is bounded by $C\left(t_{2}-t_{1}\right)$. We will combine the boundary terms with the corresponding terms of the other time intervals (see below).

Now we study the integral over $\left(t_{2}, T_{2}\right)$. We write $u_{\varepsilon}^{2}$ as $v_{\varepsilon}^{2}+\left(u_{\varepsilon}^{2}-v_{\varepsilon}^{2}\right)$, where $v_{\varepsilon}(t, r):=V_{\varepsilon}(t, z)$ is given by the approximation $V_{\varepsilon}$ of $U_{\varepsilon}$ defined in section 4 . The energy bound implies that the term with $\left(u_{\varepsilon}^{2}-v_{\varepsilon}^{2}\right)$ is bounded by

$$
C\left(\iint\left|U_{\varepsilon}-V_{\varepsilon}\right|^{2} d z d t\right)^{\frac{1}{2}}
$$

where integration is over the rescaled support of $\zeta$. Then Proposition 4.10 implies that this term converges to zero. We integrate the $v_{\varepsilon}^{2}$-term by parts:

$$
\begin{aligned}
\omega_{n} \int_{t_{2}}^{T_{2}} \int_{R_{0}}^{1} v_{\varepsilon}^{2} \partial_{t} f_{\varepsilon} \zeta r^{n-1} d r d t \\
=-\omega_{n} \int_{t_{2}}^{T_{2}} \int_{R_{0}}^{1} \partial_{t} v_{\varepsilon}^{2} f_{\varepsilon} \zeta r^{n-1} d r d t-\omega_{n} \int_{t_{2}}^{T_{2}} \int_{R_{0}}^{1} v_{\varepsilon}^{2} f_{\varepsilon} \partial_{t} \zeta r^{n-1} d r d t \\
\quad+\left.\omega_{n} \int_{R_{0}}^{1} v_{\varepsilon}^{2} f_{\varepsilon} \zeta r^{n-1} d r\right|_{t_{2}} ^{T_{2}} .
\end{aligned}
$$

Since $f_{\varepsilon}$ converges strongly in $L^{2}\left(\Omega_{T}\right)$ (cf. Lemma 3.5 ), $f_{\varepsilon}^{\prime}$ is uniformly in $L^{2}\left(\Omega_{T}\right)$, and $v_{\varepsilon}$ is given explicitly, we may conclude that the first term converges to

$$
\begin{aligned}
-\omega_{n} & \int_{t_{2}}^{T_{2}}\left(b^{2}-a^{2}\right)\left(-\frac{d}{d t} R^{j}(t)\right)\left(R^{j}(t)\right)^{n-1}\left(f_{0} \zeta\right)\left(t, R^{j}(t)\right) d t \\
& =-\int_{t_{2}}^{T_{2}}\left\langle\partial_{t} u_{0}^{2}, f_{0} \zeta\right\rangle d t .
\end{aligned}
$$

The details of this convergence are in the same spirit as the arguments of the proof of Prop. 5.2 presented in the Appendix. The second term converges to

$$
-\omega_{n} \int_{t_{2}}^{T_{2}} \int_{R_{0}}^{1} u_{0}^{2} f_{0} \partial_{t} \zeta r^{n-1} d r d t=-\int_{t_{2}}^{T_{2}} \int_{0}^{1} u_{0}^{2} f_{0} \partial_{t} \zeta d r d t .
$$

We combine the boundary term with the corresponding term from the intergration over $\left(t_{1}, t_{2}\right)$. The result converges to zero for almost every $t_{2}$, since $v_{\varepsilon}-\chi_{\varepsilon}$ converges to zero almost everywhere.

The same arguments apply for the integral over $\left(T_{1}, t_{1}\right)$. 
Finally, choosing a suitable sequence of points $t_{1}$ and $t_{2}$ with $t_{2}-t_{1} \rightarrow 0$ proves the theorem for any $\zeta$ with compact support in $(0, T) \times(0,1]$.

In case of an arbitrary test function $\zeta$ we approximate it through $\zeta \eta_{R_{0}}$, where $\eta_{R_{0}}$ is a smooth cut-off function with support in $B_{1}(0) \backslash B_{R_{0}}(0)$ and gradient such that $\nabla \eta_{R_{0}}$ is only non-zero in $B_{2 R_{0}} \backslash B_{R_{0}}(0)$. We find that

$$
\begin{gathered}
-\int_{0}^{T}\left\langle\partial_{t} u_{0}^{2}, f_{0} \zeta \eta_{R_{0}}\right\rangle d t-\int_{0}^{T} \int_{\Omega} u_{0}^{2} f_{0} \partial_{t}\left(\zeta \eta_{R_{0}}\right) d x d t \\
+\int_{0}^{T} \int_{\Omega} u_{0}^{2} \nabla f_{0} \cdot \nabla\left(\zeta \eta_{R_{0}}\right) d x d t=0 .
\end{gathered}
$$

Since $\nabla f_{0} \in L^{2}\left(\Omega_{T}\right)$ and $\nabla\left(\zeta \eta_{R_{0}}\right) \longrightarrow \nabla \zeta$ in $L^{2}\left(\Omega_{T}\right)$, the third term converges to the expected limit as $R_{0} \rightarrow 0$. The second term converges also, since $\eta_{R_{0}}$ does not depend on time. In order to handle the first term we use Lemma 3.8. It implies that

$$
g_{0} \int_{0}^{T} \int_{B_{2 R_{0}}}\left|\partial_{t} u_{0}\right| d x d t \leq \liminf _{\varepsilon \rightarrow 0} \int_{0}^{T} \int_{B_{2 R_{0}}}\left|\partial_{t} g\left(u_{\varepsilon}\right)\right| d x d t \leq C R_{0}^{2},
$$

and consequently $\int_{0}^{T}\left\langle\partial_{t} u_{0}^{2}, f_{0} \zeta \eta_{R_{0}}\right\rangle d t \longrightarrow \int_{0}^{T}\left\langle\partial_{t} u_{0}^{2}, f_{0}\right\rangle d t$. This finishes the proof.

Remark 6.4. The differential equation of Theorem 6.3 is a suitable distributional formulation of

$$
u_{0}^{2} \partial_{t} f_{0}-\operatorname{div}\left(u_{0}^{2} \nabla f_{0}\right)=0,
$$

together with the Neumann-condition for $f_{0}$.

If $\partial_{t} f_{0} \in L^{2}$, then this distributional formulation implies the jump condition $\left[u_{0}^{2} \nabla f_{0}\right]_{\left.\right|_{\Gamma}} \cdot \nu=0$ on the interface $\Gamma$.

Remark 6.5. It is also possible to derive the jump condition in the formal context of [RSK]. In fact, in taking the tangential component of (4.9) in [RSK], which is obtained by setting $\varphi=R e$ with $R$ being $u$ and with $e=(\cos f, \sin f)$ in our notation, and using the $\eta$-time scale of [RSK] (which is the one studied in the present paper), we arrive at

$$
\varepsilon R \partial_{t} f=\varepsilon(2 \nabla R \cdot \nabla f+R \Delta f) .
$$

The idea now is to multiply this by $R$ and to write the right hand side as a derivative:

$$
\varepsilon R^{2} \partial_{t} f=\varepsilon \operatorname{div}\left(R^{2} \nabla f\right) .
$$

Now it is a well established strategy to expand $R$ and $f$ in the interfacial region as $R(t, x)=R^{0}(t, z, s)+\varepsilon R^{1}(t, z, s)+\ldots$ and $f(t, x)=f^{0}(t, z, s)+\varepsilon f^{1}(t, z, s)+$ $\ldots$, where $z=\frac{d(t, x)}{\varepsilon}$ is the rescaled signed distance $d(t, x)= \pm \operatorname{dist}\left(x, \Gamma_{t}\right)$ to the interface $\Gamma$ at time $t$, and $s=s(t, x)$ is a tangential variable. In doing so, we assume that the variation of $R$ and $f$ in the transition region is only large in the orthogonal direction to the interface. The construction implies that to leading order in $\varepsilon$ the spatial derivative $\nabla_{x} d$ is the normal to the interface $\Gamma$ and thus independent of $z$ with $\left|\nabla_{x} d\right|^{2}=1$, whereas $\nabla s$ is tangential to the interface $\Gamma$, so that $\nabla_{x} d \cdot \nabla_{x} s=0$. Since $f$ does not jump across the interface, $f^{0}$ does not depend on $z$. We proved this in Lemma 3.4 even without the assumption of radial symmetry. Thus the leading 
order term of the left hand side is of order $\varepsilon$, so that the order 1 term of the right hand side has to vanish:

$$
\partial_{z}\left(\left(R^{0}\right)^{2}\left(\partial_{s} f^{0} \nabla_{x} s+\partial_{z} f^{1} \nabla_{x} d\right)\right) \cdot \nabla_{x} d=0 .
$$

Using the identities for $\nabla_{x} s$ and $\nabla_{x} d$, this simplifies into $\partial_{z}\left(\left(R^{0}\right)^{2} \partial_{z} f^{1}\right)=0$. Integration implies

$$
\left.\left(\left(R^{0}\right)^{2} \partial_{z} f^{1}\right)\right|_{z=-\infty} ^{z=+\infty}=0
$$

Matching the interfacial solution to some outer expansion implies that $R^{0}( \pm \infty)$ are the bulk values of $R$ from either side of the interface $\Gamma$, whereas $\partial_{z} f^{1}( \pm \infty)$ are the values of $\nabla f \cdot \nu$. Thus we find the jump condition on the interface.

\section{Conclusion}

We have studied the singular limit of a vectorial Allen-Cahn equation with a potential vanishing on concentric spheres and the order parameter $\varphi$ ranging in a half plane of $\mathbb{R}^{2}$. Assuming radial symmetry in space, we have established a priori bounds for the modulus $u$ and the polar angle $f$ of $\varphi$. In addition we have shown an error estimate for the difference between the exact solution $u$ and a properly rescaled travelling wave solution. This enabled us to rigorously determine the limit problem: the mean curvature flow for the interface and a harmonic map flow for the polar angle.

Many of the above results remain true for higher dimensional order parameters. Writing $\varphi=u e$ with a unit vector $e$, the original equation transforms into

$$
\begin{aligned}
\varepsilon \partial_{t} u-\varepsilon \Delta u+\frac{1}{\varepsilon} W^{\prime}(u) & =-\varepsilon u|\nabla e|^{2}, \\
u^{2} \partial_{t} e-\operatorname{div} u^{2} \nabla e & =e u^{2}|\nabla e|^{2} .
\end{aligned}
$$

It is again possible to show that any half space is an invariant region. Assuming that the data initially lie in one half space, we consequently obtain that $u$ never vanishes and $e$ is well defined. With this assumption we can introduce polar coordinates and derive all estimates of sections 3,4 and 5 in this higher dimensional case. In particular, we can deduce the mean curvature flow for the interfaces.

In section 6 we strongly used the fact that the harmonic map flow is a linear equation in the polar angle $\varphi$. This is no longer true in the higher dimensional case. One has to deal either with the nonlinear term $e u^{2}|\nabla e|^{2}$ or (after multiplication by a suitable tangent vector) with products of the form $u^{2} \partial_{t} e^{i} e^{j}$. Since we have only very little control on the time derivative of $e$, it has not been possible to pass to the limit in any of these terms.

\section{Appendix. Proof of Proposition 5.2}

Proof of Proposition 5.2. We write the differential equation (E2) in the rescaled variable $z$ :

$$
\varepsilon \partial_{t} U_{\varepsilon}-\frac{n-1}{\varepsilon z} U_{\varepsilon}^{\prime}=\frac{1}{\varepsilon}\left(U_{\varepsilon}^{\prime \prime}-W^{\prime}\left(U_{\varepsilon}\right)\right)-\frac{1}{\varepsilon} U_{\varepsilon}\left|F_{\varepsilon}^{\prime}\right|^{2} .
$$

We multiply this by $U_{\varepsilon}^{\prime}$ and integrate over $(\alpha, \beta):=\left(z_{\varepsilon}^{k}(t)+\frac{\delta}{4 \varepsilon}, z_{\varepsilon}^{m_{0}+k-1}(t)-\frac{\delta}{4 \varepsilon}\right)$, where $\delta$ is as in Definition 5.1. We multiply the result by a smooth test function of 
time $\xi$ with compact support in $B$ :

$$
\begin{aligned}
& \int_{B} \varepsilon \xi \int_{\alpha}^{\beta} \partial_{t} U_{\varepsilon} U_{\varepsilon}^{\prime} d z d t-\int_{B} \xi \int_{\alpha}^{\beta} \frac{n-1}{\varepsilon z}\left|U_{\varepsilon}^{\prime}\right|^{2} d z d t \\
& =\left.\int_{B} \xi \frac{1}{\varepsilon}\left(\frac{1}{2}\left|U_{\varepsilon}^{\prime}\right|^{2}-W\left(U_{\varepsilon}\right)\right)\right|_{\alpha} ^{\beta} d t-\int_{B} \xi \frac{1}{\varepsilon} \int_{\alpha}^{\beta} U_{\varepsilon} U_{\varepsilon}^{\prime}\left|F_{\varepsilon}^{\prime}\right|^{2} d z d t .
\end{aligned}
$$

We claim that the limit of the right hand side vanishes. Indeed, the first term on the right hand side converges to 0 because Proposition 4.10 implies that at $\alpha$ and $\beta$ we may replace $U_{\varepsilon}$ by $V_{\varepsilon}$, thereby only making an error of order $\varepsilon \sqrt{\varepsilon}$. We rewrite the second term on the right hand side in the original variable, then integrate the result by parts and use the differential equation $(E 3)$ :

$$
\begin{aligned}
\frac{1}{\varepsilon} \int_{\alpha}^{\beta} U_{\varepsilon} U_{\varepsilon}^{\prime}\left|F_{\varepsilon}^{\prime}\right|^{2} d z & =\varepsilon \int u_{\varepsilon} u_{\varepsilon}^{\prime}\left|f_{\varepsilon}^{\prime}\right|^{2} d r=\varepsilon \int\left(u_{\varepsilon}^{2} f_{\varepsilon}^{\prime}\right)^{\prime} f_{\varepsilon}^{\prime} d r-\left.\frac{\varepsilon}{2} u_{\varepsilon}^{2} f_{\varepsilon}^{\prime}\right|_{. .} ^{.} \\
& =\varepsilon \int u_{\varepsilon}^{2} \partial_{t} f_{\varepsilon} f_{\varepsilon}^{\prime} d r-\int u_{\varepsilon}^{2}\left(f_{\varepsilon}^{\prime}\right)^{2} \frac{n-1}{r} d r-\left.\frac{\varepsilon}{2} u_{\varepsilon}^{2} f_{\varepsilon}^{\prime}\right|_{. .} ^{. .}
\end{aligned}
$$

where integration is over $\left(r_{\varepsilon}^{m_{0}+k-1}(t)-\frac{\delta}{4}, r_{\varepsilon}^{k}(t)+\frac{\delta}{4}\right)$. Thus the second term of the right hand side of $(*)$ converges to 0 as a consequence of the energy estimate and Remark 3.7.

The second term on the left hand side of $(*)$ will give the curvature term. First we may replace $U_{\varepsilon}$ by $V_{\varepsilon}$, because

$$
\begin{aligned}
& \int_{B} \int_{\alpha}^{\beta}\left|\left(U_{\varepsilon}^{\prime}\right)^{2}-\left(V_{\varepsilon}^{\prime}\right)^{2}\right| d z d t \\
& \quad \leq\left(\int_{B} \int_{\alpha}^{\beta}\left|U_{\varepsilon}^{\prime}-V_{\varepsilon}^{\prime}\right| d z d t\right)^{\frac{1}{2}}\left(\int_{B} \int_{\alpha}^{\beta}\left|U_{\varepsilon}^{\prime}+V_{\varepsilon}^{\prime}\right| d z d t\right)^{\frac{1}{2}}
\end{aligned}
$$

and by the estimate of Proposition 4.1. We introduce the following notation:

$$
\begin{aligned}
(\alpha, \beta)= & \left(\alpha, \frac{z_{\varepsilon}^{m_{0}+k-2}+z_{\varepsilon}^{m_{0}+k-1}}{2}-1\right) \cup \bigcup_{i=k-1}^{m_{0}+k-1}\left(\frac{z_{\varepsilon}^{i}+z_{\varepsilon}^{i+1}}{2}-1, \frac{z_{\varepsilon}^{i}+z_{\varepsilon}^{i+1}}{2}+1\right) \\
& \cup \bigcup_{i=k-1}^{m_{0}+k-2}\left(\frac{z_{\varepsilon}^{i}+z_{\varepsilon}^{i+1}}{2}+1, \frac{z_{\varepsilon}^{i-1}+z_{\varepsilon}^{i}}{2}-1\right) \cup\left(\frac{z_{\varepsilon}^{k}+z_{\varepsilon}^{k+1}}{2}+1, \beta\right) \\
= & : T_{m_{0}+k-1} \cup \bigcup_{i=k-1}^{m_{0}+k-1} C_{i} \cup \bigcup_{i=k-1}^{m_{0}+k-2} T_{i} \cup T_{k} .
\end{aligned}
$$

Thus in $T_{i}$ the solution $V_{\varepsilon}$ is well approximated by the stationary wave solution, whereas $C_{i}$ is the region where two stationary wave solutions are matched together. We divide the integral over $(\alpha, \beta)$ as above. Then the integral over any $C_{i}$ gives 0 in the limit, because $V_{\varepsilon}^{i}$ converges pointwise to 0 in $C_{i}$ and is uniformly bounded. In any of the $T_{i}$ we may replace $\frac{n-1}{\varepsilon z}$ by $\frac{n-1}{r_{\varepsilon}^{i}(t)}$, and since $r_{\varepsilon}^{i}$ converges strongly to $\bar{r}^{i}$, and $\int_{T_{i}}\left|V_{\varepsilon}^{\prime}\right|^{2} d z$ converges strongly to the constant $g_{0}$, the limit of the second term of the left hand side of $(*)$ is

$$
-\int_{B} \xi(t) g_{0} \sum_{i=k}^{m_{0}+k-1} \frac{n-1}{\bar{r}^{i}(t)} d t .
$$


In the first term of the left hand side of $(*)$, the strategy is to replace $U_{\varepsilon}$ by $V_{\varepsilon}$ everywhere, thereby only making small errors. We first replace $U_{\varepsilon}^{\prime}$ by $V_{\varepsilon}^{\prime}$, which is possible due to the energy bound and Proposition 4.10. We integrate the result by parts with respect to time. Clearly the term involving $\partial_{t} \xi$ converges to 0 , and so does the term involving $\dot{\beta}$ and $\dot{\alpha}$, because $V_{\varepsilon}^{\prime}$ is exponentially close to 0 at $\alpha$ and $\beta$. So we are left with determining the limit of $\int_{B} \varepsilon \xi \int_{\alpha}^{\beta} U_{\varepsilon} \partial_{t} V_{\varepsilon}^{\prime} d z d t$. We integrate this by parts with respect to $z$. The resulting boundary term converges to 0 because $V_{\varepsilon}^{\prime}$ is exponentially small at $\alpha$ and $\beta$. In the remaining term we may replace $U_{\varepsilon}^{\prime}$ by $V_{\varepsilon}^{\prime}$, as a consequence of Proposition 4.1.

In order to calculate the limit of $\int_{B} \varepsilon \xi \int_{\alpha}^{\beta} V_{\varepsilon} \partial_{t} V_{\varepsilon} d z d t$, we divide the integral over $(\alpha, \beta)$ again according to the above partition. We find that

$$
\begin{aligned}
\int_{B} \varepsilon \xi \int_{T_{i}} V_{\varepsilon}^{\prime} \partial_{t} V_{\varepsilon} d z d t & =-\int_{B} \xi \frac{d}{d t} r_{\varepsilon}^{i} \int_{T_{i}}\left|\left(V_{\varepsilon}^{i}\right)^{\prime}\right|^{2} d z d t \\
& \longrightarrow-\int_{B} \xi g_{0} \frac{d}{d t} \bar{r}^{i} d t,
\end{aligned}
$$

because $\frac{d}{d t} r_{\varepsilon}^{i}$ converges weakly to $\frac{d}{d t} \bar{r}^{i}$, and $\int_{T_{i}}\left|\left(V_{\varepsilon}^{i}\right)^{\prime}\right|^{2} d z$ converges strongly to $g_{0}$. For $z \in C_{i}$ we find the formula $V_{\varepsilon}=\Xi_{\varepsilon}^{i} V_{\varepsilon}^{i}+\Xi_{\varepsilon}^{i+1} V_{\varepsilon}^{i+1}=\Xi_{\varepsilon}^{i}\left(V_{\varepsilon}^{i}-V_{\varepsilon}^{i+1}\right)+V_{\varepsilon}^{i+1}$, and thus

$$
\varepsilon \partial_{t} V_{\varepsilon}=-\Xi_{\varepsilon}^{i}\left(V_{\varepsilon}^{i}\right)^{\prime} \frac{d}{d t} r_{\varepsilon}^{i}-\Xi_{\varepsilon}^{i+1}\left(V_{\varepsilon}^{i+1}\right)^{\prime} \frac{d}{d t} r_{\varepsilon}^{i+1}-\left(\Xi_{\varepsilon}^{i}\right)^{\prime} \frac{1}{2} \frac{d}{d t}\left(r_{\varepsilon}^{i}+r_{\varepsilon}^{i+1}\right)\left(V_{\varepsilon}^{i}-V_{\varepsilon}^{i+1}\right) .
$$

Thus $\int_{C_{i}}\left|\varepsilon \partial_{t} V_{\varepsilon}\right|^{2} d z$ converges strongly to 0 , because in the partition region $B \times C_{i}$ the difference $V_{\varepsilon}^{i}-V_{\varepsilon}^{i+1}$ and the derivatives $\left(V_{\varepsilon}^{i}\right)^{\prime}$ and $\left(V_{\varepsilon}^{i+1}\right)$ converge strongly to 0 in $L^{2}$, whereas the $\frac{d}{d t} r_{\varepsilon}^{i}$ are uniformly bounded in $L^{2}(B)$. This implies the proposition.

\section{ACKNOWLEDGMENT}

This work was partially supported by the Army Research Office and the National Science Foundation through the Center for Nonlinear Analysis, the Canadian Research Council (NSERC) and the German Research Foundation (DFG) through the SFB256.

\section{REFERENCES}

[AL] H.W. Alt and St. Luckhaus (1983), Quasilinear Elliptic-Parabolic-Differential Equations, Math.Z. 183, 311-341. MR 85e:35059

[BF] M. Berger and L. Fraenkel (1970), On the Asymptotic Solution of a Nonlinear Dirichlet Problem, J. Math. Mech. 19, 553-585. MR 40:6030

[BK1] L. Bronsard and R. Kohn (1990), On the Slowness of Phase Boundary Motion in One Space Dimension, Comm. on Pure Appl. Math. 43, 983-997. MR 91f:35023

[BK2] L. Bronsard and R. Kohn (1991), Motion by Mean Curvature as the Singular Limit of Ginzburg-Landau Dynamics, J. Diff. Eq. 90, No. 2, 211-237. MR 92d:35037

[BR] L. Bronsard and F. Reitich (1993), On Three-Phase Boundary Motion and the Singular Limit of a Vector-Valued Ginzburg-Landau Equation, Arch. Rat. Mech. Anal. 124, 355379. MR 94h:35122

[BSt] L. Bronsard and B. Stoth (1997), Volume Preserving Mean Curvature Flow as a Limit of a Nonlocal Ginzburg-Landau Equation, SIAM J. Math. Anal. 28, 769-807. CMP 97:13

[C] Y. Chen (1989), Weak solutions to the evolution problem for harmonic maps into sphere, Math.Z. 201, 69-74. MR 90i:58030

[CS] Y. Chen and M. Struwe (1989), Existence and Partial Regularity Results for the Heat Flow for Harmonic Maps, Math.Z. 201, 83-103. MR 90i:58031 
[Lu] St. Luckhaus (1990), Solutions of the Two Phase Stefan Problem with the Gibbs-Thomson Law for the Melting Temperature, Europ. J. Appl. Math. 1, 101-111. MR 92i:80004

[RSK] J. Rubinstein, P. Sternberg and J. Keller (1989), Reaction-Diffusion Processes and Evolution to Harmonic Maps, SIAM J. Appl. Math. 49, 1722-1733. MR 91c:35071

[S1] P. Sternberg (1988), The effect of a singular perturbation on nonconvex variational problems, Arch. Rat. Mech. Anal. 101, 209-260. MR 89h:49007

[S2] P. Sternberg (1991), Vector-valued local minimizers of nonconvex variational problems, Rocky Mt. J. Math. 21, 799-807. MR 92e:49016

[St1] B. Stoth (1996), A Sharp Interface Limit of the Phase Field Equations: One-dimensional Axisymmetric, European J. Appl. Math. 7 (1996), 603-633. CMP 97:06

[St2] B. Stoth (1996), Convergence of the Cahn-Hilliard Equation to the Mullins-Sekerka Problem in Spherical Symmetry, J. Diff. Eq. 125, 154-183. MR 97c:35084

Department of Mathematics, McMaster University, Hamilton, Ont. L8S 4K1, Canada

E-mail address: bronsard@math.mcmaster.ca

IAM, Universität Bonn, 53115 Bonn, Deutschland

E-mail address: bstoth@iam.uni-bonn.de 